\title{
Asymptotic behavior and existence of solutions for singular elliptic equations
}

\author{
Riccardo Durastanti ${ }^{1}$ (D)
}

Received: 25 February 2019 / Accepted: 11 September 2019 / Published online: 18 September 2019 (c) Fondazione Annali di Matematica Pura ed Applicata and Springer-Verlag GmbH Germany, part of Springer Nature 2019

\section{Abstract}

We study the asymptotic behavior, as $\gamma$ tends to infinity, of solutions for the homogeneous Dirichlet problem associated with singular semilinear elliptic equations whose model is

$$
-\Delta u=\frac{f(x)}{u^{\gamma}} \text { in } \Omega,
$$

where $\Omega$ is an open, bounded subset of $\mathbb{R}^{N}$ and $f$ is a bounded function. We deal with the existence of a limit equation under two different assumptions on $f$ : either strictly positive on every compactly contained subset of $\Omega$ or only nonnegative. Through this study, we deduce optimal existence results of positive solutions for the homogeneous Dirichlet problem associated with

$$
-\Delta v+\frac{|\nabla v|^{2}}{v}=f \text { in } \Omega
$$

Keywords Semilinear elliptic equations · Quasilinear elliptic equations · Singular elliptic equations $\cdot$ Singular natural growth gradient terms $\cdot$ Asymptotic behavior

Mathematics Subject Classification 35B40 $\cdot 35 \mathrm{~J} 25 \cdot 35 \mathrm{~J} 61 \cdot 35 \mathrm{~J} 62 \cdot 35 \mathrm{~J} 75$

\section{Contents}

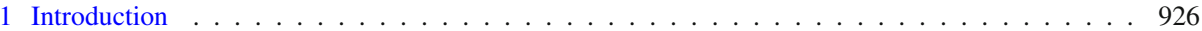

2 Main assumptions and statement of the results . . . . . . . . . . . . . . . . . . 928

3 Estimates from above and from below . . . . . . . . . . . . . . . . . . . . . 931

4 Proofs of Theorems 2.2 and $2.3 \ldots \ldots \ldots \ldots \ldots \ldots$

5 One-dimensional solutions and Proof of Theorem $2.6 \ldots \ldots \ldots \ldots$. . . . . . . . . . . . 940

6 Proof of Theorem $2.7 \ldots \ldots \ldots \ldots$. . . . . . . . . . . . . . . . . . . . . . . 948

7 Open problems . . . . . . . . . . . . . . . . . . . . . . . . 952

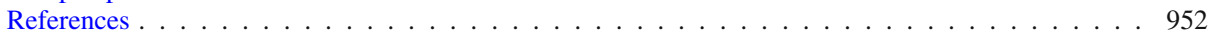

$\triangle$ Riccardo Durastanti

riccardo.durastanti@sbai.uniroma1.it

1 Dipartimento di Scienze di Base e Applicate per l' Ingegneria, "Sapienza” Università di Roma, Via Scarpa 16, 00161 Rome, Italy 


\section{Introduction}

In recent years, existence, uniqueness and regularity of nonnegative solutions of the following semilinear singular problem have been widely studied:

$$
\begin{cases}-\Delta u=\frac{f}{u^{\gamma}} & \text { in } \Omega, \\ u=0 & \text { on } \partial \Omega .\end{cases}
$$

Here, $\Omega$ is an open bounded subset of $\mathbb{R}^{N}$, with $N>2, f$ is a nonnegative function belonging to some Lebesgue space and $\gamma>0$.

Existence and uniqueness of a classical solution $u \in C^{2}(\Omega) \cap C(\bar{\Omega})$ of (1.1) are proved in $[19,39]$, when $f$ is a positive Hölder continuous function in $\bar{\Omega}$ and $\Omega$ is a smooth domain. In the same framework, Lazer and McKenna in [29] prove that $u \in W_{0}^{1,2}(\Omega)$ if and only if $\gamma<3$ and that if $\gamma>1$, the solution does not belong to $C^{1}(\bar{\Omega})$, while in [23], under the weaker assumption that $f$ is only nonnegative and bounded, Del Pino proves existence and uniqueness of a positive distributional solution belonging to $C^{1}(\Omega) \cap C(\bar{\Omega})$. These results are generalized by Lair and Shaker in [28].

Existence of a positive distributional solution with data merely in $L^{1}(\Omega)$ is proved by Boccardo and Orsina in [8]. The authors show that this solution, if $\gamma<1$, belongs to an homogeneous Sobolev space larger than $W_{0}^{1,2}(\Omega)$, if $\gamma=1$, it belongs to $W_{0}^{1,2}(\Omega)$ and, finally, if $\gamma>1$, it belongs to $W_{l o c}^{1,2}(\Omega)$ (see Theorem 3.1). In the last case, the boundary condition is assumed in a weaker sense, i.e., $u^{\frac{\gamma+1}{2}} \in W_{0}^{1,2}(\Omega)$.

Existence and regularity of solutions of (1.1) with data in suitable Lebesgue space or with measure data are also studied in $[11,12,17,18,26,35]$, while, in case of a nonlinear principal part, we refer to $[10,22,33]$. We underline also the study of qualitative properties of solutions of $(1.1)$ contained in $[13,24]$.

As concerns uniqueness of solutions of (1.1), the literature is more limited. If a solution belongs to $W_{0}^{1,2}(\Omega)$, uniqueness is proved in [7], while in [40] a necessary and sufficient condition in order to have $W_{0}^{1,2}(\Omega)$ solutions is shown. Moreover, we can find uniqueness results of solutions out of finite energy space in [14,25,34].

We observe that if we perform in (1.1) the change in variable

$$
v=\frac{u^{\gamma+1}}{\gamma+1},
$$

we formally transform (1.1) into the quasilinear singular equation with singular and gradient quadric lower-order term

$$
\begin{cases}-\Delta v+\frac{\gamma}{\gamma+1} \frac{|\nabla v|^{2}}{v}=f & \text { in } \Omega \\ v=0 & \text { on } \partial \Omega .\end{cases}
$$

Equation (1.2) is a particular case of the quasilinear singular equation

$$
\begin{cases}-\Delta v+B \frac{|\nabla v|^{2}}{v^{\rho}}=f & \text { in } \Omega, \\ v=0 & \text { on } \partial \Omega,\end{cases}
$$

where $B$ and $\rho$ are positive real numbers.

One usually says that the quadratic growth in $\nabla v$ of (1.3) is natural as this growth is invariant under the simple change of variable $w=F(v)$, where $F$ is a smooth function. In 
this case, Eq. (1.3) is also singular since the lower-order term is singular where the solution is zero.

Problem (1.3) has been recently studied by several authors. Existence of classical solutions is studied by Porru and Vitolo in [36], while existence of a positive solution $v \in W_{0}^{1,2}(\Omega)$ when $f$ is bounded and strictly positive on every compactly contained subset of $\Omega$ and $0<\rho \leq 1$ is contained in [2,4]. Moreover, if $0<\rho<1$, Boccardo proves in [6] existence of a positive weak solution under weaker assumptions on $f$, that is, $f$ only nonnegative and belonging to $L^{\left(\frac{2^{*}}{\rho}\right)^{\prime}}(\Omega)$.

As concerns the case $\rho \geq 1$, existence of positive weak solutions is proved in [6,32] for $B<1$ if $\rho=1$ and $f \in L^{\frac{2 N}{N+2}}(\Omega)$ is nonnegative in $\Omega$, while in [3] existence is proved for every $B>0$ and for every $\rho<2$ if the datum $f \in L^{\frac{2 N}{N+2}}(\Omega)$ is strictly positive on every compactly contained subset of $\Omega$. Moreover, existence of positive solutions in the same framework of [3], under a weaker assumption on $f$, that is, $f$ strictly positive on every compactly contained subset of a neighborhood of $\partial \Omega$, is proved in [16]. Nonexistence results for positive solutions in $W_{0}^{1,2}(\Omega)$ of $(1.3)$ are given, if $\rho>2$, in [3,43].

The study of the uniqueness of weak solutions of (1.3) is more limited in the literature. We refer to [5] where uniqueness is proved if $0<\rho \leq 1$ and to [15] for $\rho \geq 1$. We underline also the multiplicity result of weak solutions contained in [42].

Without the aim to be exhaustive, we also refer the reader to [20,27] in which the existence of solutions of (1.3) is studied also in the presence of sign-changing data, while we refer to $[9,21,41]$ for the study of $(1.3)$ in the parabolic case.

Looking at the results for (1.3), the case $B=1$ and $\rho=1$ is a borderline case, requiring a stronger assumption on the datum in order to prove existence of positive weak solutions. In this paper, we give an answer to the question whether this stronger assumption is really necessary or whether it is only technical.

From now onwards, we mean by $f$ strictly positive a function $f$ strictly positive on every compactly contained subset of $\Omega$, that is, for every subset $\omega$ compactly contained in $\Omega$ there exists a positive constant $c_{\omega}$ such that $f \geq c_{\omega}>0$ almost everywhere in $\omega$.

Since the case $B=1$ and $\rho=1$ can be seen as the limit case as $\gamma$ tends to infinity of Eq. (1.2), and since this equation is connected to Eq. (1.1), one can try to study problem (1.3), in the borderline case $B=1$ and $\rho=1$, by looking at the asymptotic behavior, as $\gamma$ tends to infinity, of the solutions of (1.1) under the assumption that $f$ is either nonnegative or strictly positive.

In this paper, we prove, if $f$ is strictly positive in $\Omega$, letting $\gamma$ tend to infinity, that there is no limit equation to (1.1) and we find a positive solution to

$$
\begin{cases}-\Delta v+\frac{|\nabla v|^{2}}{v}=f & \text { in } \Omega, \\ v=0 & \text { on } \partial \Omega,\end{cases}
$$

recovering the existence result contained in [2-4].

If we assume $f$ only nonnegative, more precisely zero in a neighborhood of $\partial \Omega$, we prove that there is a limit equation to (1.1) and we give a one-dimensional example providing that the assumption $f$ strictly positive cannot be relaxed in order to have a positive solution to (1.4) as a limit of approximations.

Our results imply that the existence results contained in [2-4,16,32] are sharp.

The plan of the paper is the following: In Sect. 2, we give the definitions of solution to our problems and we state the results that will be proved in the paper. In Sect. 3, we prove a priori estimates for the solutions of (1.1) both from above and from below, that allow us 
to pass to the limit in (1.1) and (1.2) as $\gamma$ tends to infinity. In Sect. 4, we pass to the limit in (1.1) under the two different assumptions on $f$. In Sect. 5, we pass to the limit in (1.2), in the case $f$ strictly positive, obtaining the existence of positive solutions of (1.4). In Sect. 6, we show, if $f$ is only nonnegative, the one-dimensional example of nonexistence of positive solutions to (1.4) obtained by approximation. To conclude, in Sect. 7 we present some open problems.

Notations Let $\Omega$ be an open and bounded subset of $\mathbb{R}^{N}$, with $N \geq 1$. We denote by $\partial \Omega$ its boundary, by $|A|$ the Lebesgue measure of a Lebesgue measurable subset $A$ of $\mathbb{R}^{N}$, and we define $\operatorname{diam}(\Omega)=\sup \{|x-y|: x, y \in \Omega\}$.

By $C_{c}(\Omega)$, we mean the space of continuous functions with compact support in $\Omega$ and by $C_{0}(\Omega)$ the space of continuous functions in $\Omega$ that are zero on $\partial \Omega$. Analogously, if $k \geq 1$, $C_{c}^{k}(\Omega)$ (resp. $C_{0}^{k}(\Omega)$ ) is the space of $C^{k}$ functions with compact support in $\Omega$ (resp. $C^{k}$ functions that are zero on $\partial \Omega$ ).

If no otherwise specified, we will denote by $C$ several constants whose value may change from line to line. These values will only depend on the data (for instance $C$ may depend on $\Omega, N$ ), but they will never depend on the indexes of the sequences we will introduce.

Moreover, for any $q>1, q^{\prime}$ will be the Hölder conjugate exponent of $q$, while for any $1 \leq p<N, p^{*}=\frac{N p}{N-p}$ will be the Sobolev conjugate exponent of $p$. We will also denote by $\epsilon(n)$ any quantity such that

$$
\limsup _{n \rightarrow \infty} \epsilon(n)=0 .
$$

We will use the following well-known functions defined for a fixed $k>0$

$$
T_{k}(s)=\max (-k, \min (s, k)) \text { and } G_{k}(s)=(|s|-k)^{+} \operatorname{sign}(s),
$$

with $s \in \mathbb{R}$.

We also mention the definition of the Gamma function

$$
\Gamma(z)=\int_{0}^{+\infty} t^{z-1} \mathrm{e}^{-t} \mathrm{~d} t,
$$

where $z$ is a complex number with positive real part, recalling that $\Gamma(1)=1$ and $\Gamma\left(\frac{1}{2}\right)=$ $\sqrt{\pi}$.

Finally, we define $\phi_{\lambda}: \mathbb{R} \rightarrow \mathbb{R}$, with $\lambda>0$, the following function

$$
\phi_{\lambda}(s)=s \mathrm{e}^{\lambda s^{2}} .
$$

In what follows we will use that for every $a, b>0$ we have, if $\lambda>\frac{b^{2}}{4 a^{2}}$, that

$$
a \phi_{\lambda}^{\prime}(s)-b\left|\phi_{\lambda}(s)\right| \geq \frac{a}{2} .
$$

\section{Main assumptions and statement of the results}

Let $M(x)$ be a matrix which satisfies, for some positive constants $0<\alpha \leq \beta$, for almost every $x \in \Omega$ and for every $\xi \in \mathbb{R}^{N}$ the following assumptions:

$$
M(x) \xi \cdot \xi \geq \alpha|\xi|^{2} \quad \text { and } \quad|M(x)| \leq \beta
$$


Let $\gamma>0$ be a real number. We consider the following semilinear elliptic problem with a singular nonlinearity

$$
\begin{cases}-\operatorname{div}(M(x) \nabla u)=\frac{f}{u^{\gamma}} & \text { in } \Omega, \\ u>0 & \text { in } \Omega, \\ u=0 & \text { on } \partial \Omega .\end{cases}
$$

To deal with existence for solutions to problem (2.2), we give the following definition of distributional solution contained in [8].

Definition 2.1 A function $u$ in $W_{l o c}^{1,1}(\Omega)$ such that

$$
\begin{cases}u \in W_{0}^{1,1}(\Omega) & \text { if } \gamma<1, \\ u^{\frac{\gamma+1}{2}} \in W_{0}^{1,1}(\Omega) & \text { if } \gamma \geq 1\end{cases}
$$

is a distributional solution of (2.2) if the following conditions are satisfied:

$$
\forall \omega \subset \subset \Omega \exists c_{\omega, \gamma}: u \geq c_{\omega, \gamma}>0 \text { in } \omega,
$$

and

$$
\int_{\Omega} M(x) \nabla u \cdot \nabla \varphi=\int_{\Omega} \frac{f \varphi}{u^{\gamma}}, \quad \forall \varphi \in C_{c}^{1}(\Omega)
$$

We underline that, if $\gamma>1$, the condition $u^{\frac{\gamma+1}{2}} \in W_{0}^{1,1}(\Omega)$ gives meaning to the boundary condition of (2.2).

We start studying the asymptotic behavior of the sequence $\left\{u_{n}\right\}$ of solutions to problem (2.2), with $\gamma=n$. Our results are the following:

Theorem 2.2 Let $f$ be a nonnegative $L^{\infty}(\Omega)$ function. Suppose that there exists $\omega \subset \subset \Omega$ such that $f=0$ in $\Omega \backslash \omega$, and such that for every $\omega^{\prime} \subset \subset \omega$ there exists $c_{\omega^{\prime}}>0$ such that $f \geq c_{\omega^{\prime}}$ in $\omega^{\prime}$. Let $\left\{u_{n}\right\}$ be a sequence of distributional solutions of

$$
\begin{cases}-\operatorname{div}\left(M(x) \nabla u_{n}\right)=\frac{f(x)}{u_{n}^{n}} & \text { in } \Omega, \\ u_{n}=0 & \text { on } \partial \Omega .\end{cases}
$$

Then, $\left\{u_{n}\right\}$ is bounded in $L^{\infty}(\Omega)$, so that it converges, up to subsequences, to a bounded function $u$ which is identically equal to 1 almost everywhere in $\omega$. Furthermore, the sequence $\left\{f(x) / u_{n}^{n}\right\}$ is bounded in $L^{1}(\Omega)$, and if $\mu$ is the $*$-weak limit in the sense of measures of $f(x) / u_{n}^{n}$, $\mu$ is concentrated on $\partial \omega$, and $u$ in $W_{0}^{1,2}(\Omega)$ is the solution of

$$
\begin{cases}-\operatorname{div}(M(x) \nabla u)=\mu & \text { in } \Omega \\ u=0 & \text { on } \partial \Omega .\end{cases}
$$

Theorem 2.3 Let $f$ be a function belonging to $L^{\infty}(\Omega)$ such that for every $\omega \subset \subset \Omega$ there exists $c_{\omega}>0$ such that $f \geq c_{\omega}$ in $\omega$. Let $\left\{\omega_{n}\right\}$ be an increasing sequence of compactly contained subsets of $\Omega$ such that their union is $\Omega$, and let $u_{n}$ be the distributional solution of

$$
\begin{cases}-\operatorname{div}\left(M(x) \nabla u_{n}\right)=\frac{f(x) \chi_{\omega_{n}}}{u_{n}^{n}} & \text { in } \Omega, \\ u_{n}=0 & \text { on } \partial \Omega .\end{cases}
$$


Then, $\left\{u_{n}\right\}$ is bounded in $L^{\infty}(\Omega)$, so that it converges, up to subsequences, to a bounded function $u$, which is identically equal to 1 almost everywhere in $\Omega$. Moreover, the sequence $\left\{f(x) \chi_{\omega_{n}} / u_{n}^{n}\right\}$ is unbounded in $L^{1}(\Omega)$, and there is no limit equation for $u$.

If $M(x) \equiv I$, we have that $\left\{v_{n}=\frac{u_{n}^{n+1}}{n+1}\right\}$ is a sequence of distributional solutions to the following problem

$$
\begin{cases}-\Delta v_{n}+\frac{n}{n+1} \frac{\left|\nabla v_{n}\right|^{2}}{v_{n}}=f(x) & \text { in } \Omega \\ v_{n}=0 & \text { on } \partial \Omega .\end{cases}
$$

To be complete, we give the definitions of distributional and weak solution for quasilinear elliptic equations with singular and gradient quadratic lower-order term whose model is

$$
\begin{cases}-\Delta v+B \frac{|\nabla v|^{2}}{v}=f & \text { in } \Omega \\ v=0 & \text { on } \partial \Omega\end{cases}
$$

where $B>0$.

Definition 2.4 A function $v$ in $W_{0}^{1,2}(\Omega)$ is a weak solution of (2.7) if the following conditions are satisfied:

(i) $v>0$ almost everywhere in $\Omega$,

(ii) $\frac{|\nabla v|^{2}}{v}$ belongs to $L^{1}(\Omega)$,

(iii) It holds

$$
\int_{\Omega} \nabla v \cdot \nabla \varphi+B \int_{\Omega} \frac{|\nabla v|^{2}}{v} \varphi=\int_{\Omega} f \varphi, \quad \forall \varphi \in W_{0}^{1,2}(\Omega) \cap L^{\infty}(\Omega) .
$$

Definition 2.5 A function $v$ in $W_{0}^{1,1}(\Omega)$ is a distributional solution of (2.7) if the following conditions are satisfied:

(i) $v>0$ almost everywhere in $\Omega$,

(ii) $\frac{|\nabla v|^{2}}{v}$ belongs to $L^{1}(\Omega)$,

(iii) It holds

$$
\int_{\Omega} \nabla v \cdot \nabla \varphi+B \int_{\Omega} \frac{|\nabla v|^{2}}{v} \varphi=\int_{\Omega} f \varphi, \quad \forall \varphi \in C_{c}^{1}(\Omega) .
$$

By passing to the limit in (2.6), we prove, in the case $f$ strictly positive, the following existence theorem of weak solution to problem (2.8).

Theorem 2.6 Let $f$ be a $L^{\infty}(\Omega)$ function such that for every $\omega \subset \subset \Omega$ there exists $c_{\omega}>0$ such that $f \geq c_{\omega}$ in $\omega$. Then, $\left\{v_{n}=\frac{u_{n}^{n}+1}{n+1}\right\}$ is bounded in $W_{0}^{1,2}(\Omega) \cap L^{\infty}(\Omega)$, so that it converges, up to subsequences, to a bounded nonnegative function $v$ which is a weak solution of

$$
\begin{cases}-\Delta v+\frac{|\nabla v|^{2}}{v}=f & \text { in } \Omega \\ v=0 & \text { on } \partial \Omega\end{cases}
$$


On the other hand, if $f$ is zero in a neighborhood of $\partial \Omega$, we show by a one-dimensional explicit example that the function obtained as limit of our approximation is zero in a subset of $\Omega$ with strictly positive measure. In other words, we will prove the following result.

Theorem 2.7 Let $\Omega=(-2,2)$ and $\omega=(-1,1)$. Let $u_{n}$ in $W_{0}^{1,2}((-2,2))$ be the weak solution of

$$
\left\{\begin{array}{l}
-u_{n}^{\prime \prime}(t)=\frac{\chi_{(-1,1)}}{u_{n}^{n}} \text { in }(-2,2) \\
u_{n}( \pm 2)=0
\end{array}\right.
$$

Let $v_{n}=\frac{u_{n}^{n+1}}{n+1}$ be a weak solution of

$$
\left\{\begin{array}{l}
-v_{n}^{\prime \prime}+\frac{n}{n+1} \frac{\left|v_{n}^{\prime}\right|^{2}}{v_{n}}=\chi_{(-1,1)} \text { in }(-2,2) \\
v_{n}( \pm 2)=0
\end{array}\right.
$$

then $\left\{v_{n}\right\}$ weakly converges to a function $v$ in $W_{0}^{1,2}((-2,2))$ and $v$, belonging to $C_{0}^{\infty}((-1,1))$, is a classical solution of

$$
\left\{\begin{array}{l}
-v^{\prime \prime}+\frac{\left|v^{\prime}\right|^{2}}{v}=1 \text { in }(-1,1) \\
v( \pm 1)=0
\end{array}\right.
$$

Moreover, $v(t)=\frac{2}{\pi^{2}} \cos ^{2}\left(\frac{\pi}{2} t\right)$ in $(-1,1)$ and $v(t) \equiv 0$ in $[-2,-1] \cup[1,2]$.

Remark 2.8 It follows from Theorem 2.7 that if $f$ is only nonnegative we cannot obtain by approximation a positive solution of (2.8). This implies that the assumption $f$ strictly positive is necessary (and not only technical) to have positive solutions on the whole $\Omega$ to problem (2.8). Hence, the existence results contained in [2-4,16,32] are optimal.

\section{Estimates from above and from below}

In [8], existence results for distributional solutions of (2.2) have been proved. To be more precise, we have the following theorem in the case $\gamma>1$.

Theorem 3.1 Let $\gamma>1$, and let $f$ be in $L^{\infty}(\Omega)$, with $f \geq 0$ in $\Omega$, $f$ not identically zero. Then, there exists a distributional solution $u$ of (2.2), with $\mathrm{u}$ in $W_{\mathrm{loc}}^{1,2}(\Omega) \cap L^{\infty}(\Omega)$. Moreover, we can extend the class of test functions in the sense that

$$
\int_{\Omega} M(x) \nabla u \cdot \nabla \varphi=\int_{\Omega} \frac{f \varphi}{u^{\gamma}}, \quad \forall \varphi \in W_{0}^{1,2}(\Omega) \text { with compact support. }
$$

Sketch of the Proof of Theorem 3.1 Following [8], let $m$ in $\mathbb{N}$ and consider the approximated problems

$$
\begin{cases}-\operatorname{div}\left(M(x) \nabla u_{m}\right)=\frac{f}{\left(u_{m}+\frac{1}{m}\right)^{\gamma}} & \text { in } \Omega, \\ u_{m}>0 & \text { in } \Omega, \\ u_{m}=0 & \text { on } \partial \Omega .\end{cases}
$$


The existence of a solution $u_{m}$ can be easily proved by means of the Schauder fixed point theorem. Since the sequence $g_{m}(s)=\frac{1}{\left(s+\frac{1}{m}\right)^{\gamma}}$ is increasing in $m$, standard elliptic estimates imply that the sequence $\left\{u_{m}\right\}$ is increasing, so that $u_{m} \geq u_{1}$, and there exists the pointwise limit $u$ of $u_{m}$. Since (by the maximum principle) for every $\omega \subset \subset \Omega$ there exists $c_{\omega, \gamma}>0$ such that $u_{1} \geq c_{\omega, \gamma}$ in $\omega$, it then follows that $u_{m}$ (and so $u$ ) has the same property.

Choosing $u_{m}^{\gamma}$ as test function in (3.2), we obtain, using (2.1), that

$$
\frac{4 \alpha \gamma}{(\gamma+1)^{2}} \int_{\Omega}\left|\nabla u_{m}^{\frac{\gamma+1}{2}}\right|^{2} \leq \gamma \int_{\Omega} M(x) \nabla u_{m} \cdot \nabla u_{m} u_{m}^{\gamma-1}=\int_{\Omega} \frac{f u_{m}^{\gamma}}{\left(u_{m}+\frac{1}{m}\right)^{\gamma}} \leq \int_{\Omega} f .
$$

Therefore, $\left\{u_{m}^{\frac{\gamma+1}{2}}\right\}$ is bounded in $W_{0}^{1,2}(\Omega)$. Choosing $u_{m} \varphi^{2}$ as test function in (3.2), with $\varphi$ in $C_{0}^{1}(\Omega)$, we obtain, using again $(2.1)$,

$$
\alpha \int_{\Omega}\left|\nabla u_{m}\right|^{2} \varphi^{2}+2 \int_{\Omega} M(x) \nabla u_{m} \nabla \varphi u_{m} \varphi \leq \int_{\Omega} \frac{f u_{m} \varphi^{2}}{\left(u_{m}+\frac{1}{m}\right)^{\gamma}} .
$$

Hence, if $\omega=\{\varphi \neq 0\}$, recalling that $u_{m} \geq c_{\omega, \gamma}>0$ in $\omega$, we have, by Young's inequality,

$$
\alpha \int_{\Omega}\left|\nabla u_{m}\right|^{2} \varphi^{2} \leq \frac{\alpha}{2} \int_{\Omega}\left|\nabla u_{m}\right|^{2} \varphi^{2}+C \int_{\Omega}|\nabla \varphi|^{2} u_{m}^{2}+\frac{\left\|f \varphi^{2}\right\|_{L^{\infty}(\Omega)}}{c_{\omega, \gamma}^{\gamma}} \int_{\Omega} u_{m} .
$$

Since $u_{m}$ is bounded in $L^{2}(\Omega)$ (recall that $u_{m}^{\frac{\gamma+1}{2}}$ is bounded in $W_{0}^{1,2}(\Omega)$, so that $u_{m}^{\gamma+1}$ is bounded in $L^{1}(\Omega)$ by Poincaré inequality, and that $\gamma>1$ ), we thus have

$$
\int_{\Omega}\left|\nabla u_{m}\right|^{2} \varphi^{2} \leq C
$$

so that the sequence $\left\{u_{m}\right\}$ is bounded in $W_{\text {loc }}^{1,2}(\Omega)$. Let now $k>1$ and choose $G_{k}\left(u_{m}\right)$ as test function in (3.2). We obtain, using (2.1),

$$
\alpha \int_{\Omega}\left|\nabla G_{k}\left(u_{m}\right)\right|^{2} \leq \int_{\Omega} \frac{f G_{k}\left(u_{m}\right)}{\left(u_{m}+\frac{1}{m}\right)^{\gamma}} \leq \frac{1}{k^{\gamma}} \int_{\Omega} f G_{k}\left(u_{m}\right),
$$

so that

$$
\alpha \int_{\Omega}\left|\nabla G_{k}\left(u_{m}\right)\right|^{2} \leq \int_{\Omega} f G_{k}\left(u_{m}\right), \quad \forall k \geq 1 .
$$

Starting from this inequality, and reasoning as in Theorem 4.2 of [37], we can prove that $u_{m}$ is uniformly bounded in $L^{\infty}(\Omega)$, so that $u$ belongs to $L^{\infty}(\Omega)$ as well.

Once we have the a priori estimates on $u_{m}$, we can pass to the limit in the approximate equation with test functions $\varphi$ in $W_{0}^{1,2}(\Omega)$ with compact support; indeed,

$$
\lim _{m \rightarrow+\infty} \int_{\Omega} M(x) \nabla u_{m} \cdot \nabla \varphi=\int_{\Omega} M(x) \nabla u \cdot \nabla \varphi,
$$

since $u_{m}$ is weakly convergent to $u$ in $W_{\text {loc }}^{1,2}(\Omega)$, and

$$
\lim _{m \rightarrow+\infty} \int_{\Omega} \frac{f \varphi}{\left(u_{m}+\frac{1}{m}\right)^{\gamma}}=\int_{\Omega} \frac{f \varphi}{u^{\gamma}},
$$

by the Lebesgue theorem, since $u_{m} \geq c_{\{\varphi \neq 0\}, \gamma}>0$ on the support of $\varphi$. 
Since the formulation of distributional solution for (2.2) is not suitable for our purposes, we are going to better specify the class of test functions which are admissible for the problem (2.2) to obtain estimates from above for $u$. We start with the following theorem.

Theorem 3.2 The solution $u$ of (2.2) given by Theorem 3.1 is such that:

(i) $u^{\gamma+1}$ belongs to $W_{0}^{1,2}(\Omega)$;

(ii)

$$
\int_{\Omega} M(x) \nabla\left(\frac{u^{\gamma+1}}{\gamma+1}\right) \cdot \nabla v \leq \int_{\Omega} f v, \quad \forall v \in W_{0}^{1,2}(\Omega), v \geq 0
$$

(iii)

$$
\|u\|_{L^{\infty}(\Omega)} \leq\left[C(\gamma+1)\|f\|_{L^{\infty}(\Omega)}\right]^{\frac{1}{\gamma+1}},
$$

for some constant $C>0$, independent on $\gamma$.

Proof We begin by observing that, using the boundedness in $L^{\infty}(\Omega)$ of the sequence $u_{m}$ of solutions of (3.2), and the boundedness of $u_{m}^{\frac{\gamma+1}{2}}$ in $W_{0}^{1,2}(\Omega)$, the sequence $u_{m}^{p}$ is bounded in $W_{0}^{1,2}(\Omega)$ for every $p \geq \frac{\gamma+1}{2}$. In particular, $\left\{u_{m}^{\gamma+1}\right\}$ is bounded in $W_{0}^{1,2}(\Omega)$. This yields that $u^{\gamma+1}$ belongs to $W_{0}^{1,2}(\Omega)$ as well; i.e., i) is proved.

We now fix a positive $\varphi$ in $C_{0}^{1}(\Omega)$ and take $u_{m}^{\gamma} \varphi$ as test function in (3.2). We obtain

$$
\gamma \int_{\Omega} M(x) \nabla u_{m} \cdot \nabla u_{m} u_{m}^{\gamma-1} \varphi+\int_{\Omega} M(x) \nabla u_{m} \cdot \nabla \varphi u_{m}^{\gamma} \leq \int_{\Omega} f \varphi .
$$

Dropping the first term (which is positive), we obtain

$$
\int_{\Omega} M(x) \nabla\left(\frac{u_{m}^{\gamma+1}}{\gamma+1}\right) \cdot \nabla \varphi \leq \int_{\Omega} f \varphi .
$$

Letting $m$ tend to infinity, and using the boundedness of $u_{m}^{\gamma+1}$ in $W_{0}^{1,2}(\Omega)$, we obtain

$$
\int_{\Omega} M(x) \nabla\left(\frac{u^{\gamma+1}}{\gamma+1}\right) \cdot \nabla \varphi \leq \int_{\Omega} f \varphi, \quad \forall \varphi \in C_{0}^{1}(\Omega), \varphi \geq 0 .
$$

Since $u^{\gamma+1}$ belongs to $W_{0}^{1,2}(\Omega)$, we obtain by density

$$
\int_{\Omega} M(x) \nabla\left(\frac{u^{\gamma+1}}{\gamma+1}\right) \cdot \nabla v \leq \int_{\Omega} f v, \quad \forall v \in W_{0}^{1,2}(\Omega), v \geq 0,
$$

which is (3.3). We now choose

$$
v=G_{k}\left(\frac{u^{\gamma+1}}{\gamma+1}\right)
$$

as test function in (3.3) (recall that $u \geq 0$, so that $v \geq 0$ as well). We obtain, setting $A_{\gamma}(k)=\left\{u^{\gamma+1} \geq(\gamma+1) k\right\}=\{v \geq 0\}$,

$$
\int_{A_{\gamma}(k)} M(x) \nabla\left(\frac{u^{\gamma+1}}{\gamma+1}\right) \cdot \nabla G_{k}\left(\frac{u^{\gamma+1}}{\gamma+1}\right) \leq \int_{A_{\gamma}(k)} f G_{k}\left(\frac{u^{\gamma+1}}{\gamma+1}\right) .
$$


Recalling (2.1), we therefore have

$$
\alpha \int_{A_{\gamma}(k)}\left|\nabla G_{k}\left(\frac{u^{\gamma+1}}{\gamma+1}\right)\right|^{2} \leq \int_{A_{\gamma}(k)} f G_{k}\left(\frac{u^{\gamma+1}}{\gamma+1}\right) .
$$

From this inequality, reasoning once again as in [37], we obtain that there exists $C>0$ such that

$$
\left\|\frac{u^{\gamma+1}}{\gamma+1}\right\|_{L^{\infty}(\Omega)} \leq C\|f\|_{L^{\infty}(\Omega)},
$$

which then yields (3.4).

Remark 3.3 We observe that if we also assume that $\omega=\{f>0\}$ is compactly contained in $\Omega$ in Theorem 3.1, then $u$ belongs to $W_{0}^{1,2}(\Omega)$ and $\frac{f}{u^{\gamma}}$ belongs to $L^{1}(\Omega)$. As a matter of fact, taking $u_{m}$ as test function in (3.2), we have

$$
\alpha \int_{\Omega}\left|\nabla u_{m}\right|^{2} \leq \int_{\Omega} \frac{f u_{m}}{\left(u_{m}+\frac{1}{m}\right)^{\gamma}} \leq \frac{\|f\|_{L^{\infty}(\Omega)}}{c_{\omega, \gamma}^{\gamma-1}},
$$

so that $u$ belongs to $W_{0}^{1,2}(\Omega)$. Moreover, using the Lebesgue theorem and that $u_{m} \geq c_{\omega, \gamma}$, we deduce that $\frac{f}{u_{m}^{\gamma}}$ strongly converges to $\frac{f}{u^{\gamma}}$ in $L^{1}(\Omega)$. As a consequence, we can extend the class of test functions for $(3.1)$ to $W_{0}^{1,2}(\Omega)$.

Remark 3.4 Under the assumptions of Remark 3.3, thanks to the results contained in [7], it follows that $u$ is the unique weak solution of (2.2).

From now on, $\gamma=n$, and we will denote by $u_{n}$ the solution of (2.3); therefore, by the results of Theorem 3.2, we have that $u_{n}^{n+1}$ belongs to $W_{0}^{1,2}(\Omega) \cap L^{\infty}(\Omega)$ and that

$$
\left\|u_{n}\right\|_{L^{\infty}(\Omega)} \leq\left(C(n+1)\|f\|_{L^{\infty}(\Omega)}\right)^{\frac{1}{n+1}},
$$

which in particular implies that

$$
\limsup _{n \rightarrow+\infty}\left\|u_{n}\right\|_{L^{\infty}(\Omega)} \leq 1 .
$$

We now consider the estimates from below on the sequence $\left\{u_{n}\right\}$. We first need to enunciate two technical lemmas that we will use during the proof of these estimates.

Lemma 3.5 Let $m(j, r):[0,+\infty) \times\left[0, R_{0}\right) \rightarrow[0,+\infty)$ be a function such that $m(\cdot, r)$ is nonincreasing and $m(j, \cdot)$ is nondecreasing. Moreover, suppose that there exist $k_{0} \geq 0$, $C, \nu, \delta>0$ and $\mu>1$ satisfying

$$
m(j, r) \leq C \frac{m(k, R)^{\mu}}{(j-k)^{v}(R-r)^{\delta}} \quad \forall j>k \geq k_{0}, 0 \leq r<R<R_{0} .
$$

Then, for every $0<\sigma<1$, there exists $d>0$ such that

$$
m\left(k_{0}+d,(1-\sigma) R_{0}\right)=0,
$$

where $d^{\nu}=\frac{2^{(\nu+\delta) \frac{\mu}{\mu-1}} C m\left(k_{0}, R_{0}\right)^{\mu-1}}{\sigma^{\delta} R_{0}^{\delta}}$. 
Proof See [38].

Lemma 3.6 Let $g:[0,+\infty) \rightarrow[0,+\infty)$ be a continuous and increasing function, with $g(0)=0$, such that

$$
t \in(0,+\infty) \mapsto \frac{g(t)}{t} \text { is increasing and } \int^{+\infty} \frac{1}{\sqrt{t g(t)}}<+\infty .
$$

Then, for any $C>0$ and $\delta \geq 0$, there exists a function $\varphi:[0,1] \rightarrow[0,1]$ depending on $g, C, \delta$ with $\varphi \in C^{1}([0,1]), \sqrt{\varphi} \in C^{1}([0,1]), \varphi(0)=\varphi^{\prime}(0)=0, \varphi(1)=1, \varphi(\sigma)>0$ for every $\sigma>0$ and satisfying

$$
t^{\delta+1} \frac{\varphi^{\prime}(\sigma)^{2}}{\varphi(\sigma)} \leq \frac{1}{C} t^{\delta} g(t) \varphi(\sigma)+1, \quad \forall 0 \leq \sigma \leq 1, t \geq 0 .
$$

Proof See [30], Lemma 1.1.

We are ready to prove the estimates from below.

Theorem 3.7 Let $u_{n}$ be the solution of (2.3) given by Theorem 3.1, and let $\omega \subset \subset \Omega$ be such that for every $\omega^{\prime} \subset \subset \omega$ there exists $c_{\omega^{\prime}}>0$ satisfying $f \geq c_{\omega^{\prime}}$ in $\omega^{\prime}$. Then, there exists $M_{\omega^{\prime}}>0$ such that

$$
u_{n} \geq(n+1)^{\frac{1}{n+1}} \mathrm{e}^{-\frac{M_{\omega^{\prime}}}{n+1}} \quad \text { in } \omega^{\prime}
$$

Proof Let $\omega^{\prime \prime} \subset \subset \omega^{\prime} \subset \subset \omega$, by the assumptions we have that

$$
m_{\omega^{\prime}}=\inf _{x \in \omega^{\prime}} f(x)>0 .
$$

Let $\eta$ in $C_{0}^{1}(\Omega)$ be such that

$$
\eta(x)= \begin{cases}1 & \text { in } \omega^{\prime \prime} \\ 0 & \text { in } \Omega \backslash \overline{\omega^{\prime}}\end{cases}
$$

We consider the function $\varphi \in C^{1}([0,1])$ given by Lemma 3.6, in correspondence of $g(t)=$ $\mathrm{e}^{t}-1, \delta=1$ and of an arbitrary constant $C>0$. Define

$$
\begin{aligned}
\xi(x) & =\sqrt{\varphi(\eta(x))} \in C_{0}^{1}(\Omega), \\
z_{n} & =-\log \left(\frac{u_{n}^{n+1}}{n+1}\right),
\end{aligned}
$$

and, for $k>0$,

$$
v_{n}=\frac{G_{k}\left(z_{n}^{+}\right)}{u_{n}} .
$$

Note that $v_{n} \geq 0$ is well defined, since where $z_{n}^{+}>k$ one has $u_{n} \neq 0$. We have

$$
\nabla \xi=\frac{\varphi^{\prime}(\eta)}{2 \sqrt{\varphi(\eta)}} \nabla \eta
$$

Since

$$
\nabla z_{n}=-\frac{(n+1) \nabla u_{n}}{u_{n}}
$$


we obtain

$$
\nabla v_{n}=-\frac{\nabla u_{n}}{u_{n}^{2}} G_{k}\left(z_{n}^{+}\right)+\frac{1}{u_{n}} \nabla z_{n} \chi_{A_{n}(k)}=-\frac{\nabla u_{n}}{u_{n}^{2}} G_{k}\left(z_{n}^{+}\right)-\frac{(n+1) \nabla u_{n}}{u_{n}^{2}} \chi_{A_{n}(k)},
$$

where $A_{n}(k)=\left\{z_{n}^{+} \geq k\right\}=\left\{G_{k}\left(z_{n}^{+}\right) \neq 0\right\}$. Therefore, since $u_{n}$ belongs to $W_{\text {loc }}^{1,2}(\Omega) \cap$ $L^{\infty}(\Omega)$ and it is locally positive, $z_{n}$ and $v_{n}$ belong to $W_{\text {loc }}^{1,2}(\Omega)$. Consequently, the positive function $v_{n} \xi^{2}$ belongs to $W_{0}^{1,2}(\Omega)$, has compact support and can be chosen as test function in (3.1), with $\gamma=n$, to obtain

$$
\begin{aligned}
& -\int_{A_{n}(k)} M(x) \nabla u_{n} \cdot \nabla u_{n} \frac{G_{k}\left(z_{n}^{+}\right) \xi^{2}}{u_{n}^{2}}-\int_{A_{n}(k)} M(x) \nabla u_{n} \cdot \nabla u_{n} \frac{(n+1) \xi^{2}}{u_{n}^{2}} \\
& \quad+2 \int_{A_{n}(k)} M(x) \nabla u_{n} \cdot \nabla \xi \frac{G_{k}\left(z_{n}^{+}\right) \xi}{u_{n}}=\int_{A_{n}(k)} \frac{f G_{k}\left(z_{n}^{+}\right) \xi^{2}}{u_{n}^{n+1}} .
\end{aligned}
$$

Since

$$
\frac{n+1}{u_{n}^{n+1}}=\mathrm{e}^{z_{n}}
$$

the previous identity can be rewritten as

$$
\begin{gathered}
-\frac{1}{n+1} \int_{A_{n}(k)} M(x) \nabla z_{n} \cdot \nabla z_{n} G_{k}\left(z_{n}^{+}\right) \xi^{2}-\int_{A_{n}(k)} M(x) \nabla z_{n} \cdot \nabla z_{n} \xi^{2} \\
-2 \int_{A_{n}(k)} M(x) \nabla z_{n} \cdot \nabla \xi G_{k}\left(z_{n}^{+}\right) \xi=\int_{A_{n}(k)} f \mathrm{e}^{z_{n}^{+}} G_{k}\left(z_{n}^{+}\right) \xi^{2} .
\end{gathered}
$$

Since the first term is negative, we have, using (2.1) and (3.7), as well as the fact that $G_{k}\left(s^{+}\right) \leq s^{+}$, that

$$
\alpha \int_{A_{n}(k)}\left|\nabla z_{n}\right|^{2} \xi^{2}+m_{\omega^{\prime}} \int_{A_{n}(k)} \mathrm{e}^{G_{k}\left(z_{n}^{+}\right)} G_{k}\left(z_{n}^{+}\right) \xi^{2} \leq 2 \beta \int_{A_{n}(k)}\left|\nabla z_{n}\right||\nabla \xi| G_{k}\left(z_{n}^{+}\right) \xi .
$$

Using Young's inequality in the right-hand side, we have

$$
2 \beta \int_{A_{n}(k)}\left|\nabla z_{n}\right||\nabla \xi| G_{k}\left(z_{n}^{+}\right) \xi \leq \frac{\alpha}{2} \int_{A_{n}(k)}\left|\nabla z_{n}\right|^{2} \xi^{2}+\frac{2 \beta^{2}}{\alpha} \int_{A_{n}(k)}|\nabla \xi|^{2} G_{k}\left(z_{n}^{+}\right)^{2},
$$

so that we have

$$
\frac{\alpha}{2} \int_{A_{n}(k)}\left|\nabla G_{k}\left(z_{n}^{+}\right)\right|^{2} \xi^{2}+m_{\omega^{\prime}} \int_{A_{n}(k)} \mathrm{e}^{G_{k}\left(z_{n}^{+}\right)} G_{k}\left(z_{n}^{+}\right) \xi^{2} \leq \frac{2 \beta^{2}}{\alpha} \int_{A_{n}(k)}|\nabla \xi|^{2} G_{k}\left(z_{n}^{+}\right)^{2} .
$$

Observing that

$$
\frac{\alpha}{4}\left|\nabla\left(G_{k}\left(z_{n}^{+}\right) \xi\right)\right|^{2} \leq \frac{\alpha}{2}\left|\nabla G_{k}\left(z_{n}^{+}\right)\right|^{2} \xi^{2}+\frac{\alpha}{2}|\nabla \xi|^{2} G_{k}\left(z_{n}^{+}\right)^{2},
$$

we obtain

$$
\frac{\alpha}{4} \int_{A_{n}(k)}\left|\nabla\left(G_{k}\left(z_{n}^{+}\right) \xi\right)\right|^{2}+m_{\omega^{\prime}} \int_{A_{n}(k)} \mathrm{e}^{G_{k}\left(z_{n}^{+}\right)} G_{k}\left(z_{n}^{+}\right) \xi^{2} \leq \frac{4 \beta^{2}+\alpha^{2}}{2 \alpha} \int_{A_{n}(k)}|\nabla \xi|^{2} G_{k}\left(z_{n}^{+}\right)^{2} .
$$


Using that $\xi=\sqrt{\varphi(\eta)}$ and (3.8), we deduce

$$
\begin{aligned}
& \frac{\alpha}{4} \int_{A_{n}(k)}\left|\nabla\left(G_{k}\left(z_{n}^{+}\right) \xi\right)\right|^{2}+m_{\omega^{\prime}} \int_{A_{n}(k)} \mathrm{e}^{G_{k}\left(z_{n}^{+}\right)} G_{k}\left(z_{n}^{+}\right) \varphi(\eta) \\
& \quad \leq \frac{4 \beta^{2}+\alpha^{2}}{8 \alpha}\|\nabla \eta\|_{L^{\infty}(\Omega)}^{2} \int_{A_{n}(k)} G_{k}\left(z_{n}^{+}\right)^{2} \frac{\varphi^{\prime}(\eta)^{2}}{\varphi(\eta)} .
\end{aligned}
$$

Applying Lemma 3.6, with $t=G_{k}\left(z_{n}^{+}\right)$, and choosing the constant $C$ as

$$
C=\frac{4 \beta^{2}+\alpha^{2}}{4 \alpha m_{\omega^{\prime}}}\|\nabla \eta\|_{L^{\infty}(\Omega)}^{2},
$$

we have

$$
\begin{aligned}
& \frac{4 \beta^{2}+\alpha^{2}}{8 \alpha}\|\nabla \eta\|_{L^{\infty}(\Omega)}^{2} \int_{A_{n}(k)} G_{k}\left(z_{n}^{+}\right)^{2} \frac{\varphi^{\prime}(\eta)^{2}}{\varphi(\eta)} \\
& \quad \leq \frac{m_{\omega^{\prime}}}{2} \int_{A_{n}(k)} G_{k}\left(z_{n}^{+}\right)\left(\mathrm{e}^{G_{k}\left(z_{n}^{+}\right)}-1\right) \varphi(\eta)+\frac{4 \beta^{2}+\alpha^{2}}{8 \alpha}\|\nabla \eta\|_{L^{\infty}(\Omega)}^{2}\left|A_{n}(k) \cap \omega^{\prime}\right| .
\end{aligned}
$$

Hence, we obtain

$$
\begin{aligned}
& \frac{\alpha}{4} \int_{A_{n}(k)}\left|\nabla\left(G_{k}\left(z_{n}^{+}\right) \xi\right)\right|^{2}+\frac{m_{\omega^{\prime}}}{2} \int_{A_{n}(k)} \mathrm{e}^{G_{k}\left(z_{n}^{+}\right)} G_{k}\left(z_{n}^{+}\right) \varphi(\eta) \\
& \quad+\frac{m_{\omega^{\prime}}}{2} \int_{A_{n}(k)} G_{k}\left(z_{n}^{+}\right) \varphi(\eta) \leq \frac{4 \beta^{2}+\alpha^{2}}{8 \alpha}\|\nabla \eta\|_{L^{\infty}(\Omega)}^{2}\left|A_{n}(k) \cap \omega^{\prime}\right| .
\end{aligned}
$$

Dropping the positive terms in the left-hand side, we have

$$
\int_{A_{n}(k)}\left|\nabla\left(G_{k}\left(z_{n}^{+}\right) \xi\right)\right|^{2} \leq \frac{4 \beta^{2}+\alpha^{2}}{2 \alpha^{2}}\|\nabla \eta\|_{L^{\infty}(\Omega)}^{2}\left|A_{n}(k) \cap \omega^{\prime}\right| .
$$

Moreover, denoting with $\mathcal{S}$ the constant given by the Sobolev embedding theorem and recalling that $\xi \equiv 1$ in $\omega^{\prime \prime}$, we deduce, for $j>k>0$, that

$$
\begin{aligned}
& (j-k)^{2}\left|A_{n}(j) \cap \omega^{\prime \prime}\right|^{\frac{2}{2^{*}}} \leq\left(\int_{A_{n}(j) \cap \omega^{\prime \prime}}\left|G_{k}\left(z_{n}^{+}\right)\right|^{2^{*}}\right)^{\frac{2}{2^{*}}} \\
& \quad \leq\left(\int_{A_{n}(k) \cap \omega^{\prime}}\left|G_{k}\left(z_{n}^{+}\right) \xi\right|^{2^{*}}\right)^{\frac{2}{2^{*}}} \leq \mathcal{S}^{2} \frac{4 \beta^{2}+\alpha^{2}}{2 \alpha^{2}}\|\nabla \eta\|_{L^{\infty}(\Omega)}^{2}\left|A_{n}(k) \cap \omega^{\prime}\right| .
\end{aligned}
$$

Defining $c_{0}^{\frac{2}{2^{*}}}=\mathcal{S}^{2} \frac{4 \beta^{2}+\alpha^{2}}{2 \alpha^{2}}$, we have, for all $\omega^{\prime \prime} \subset \subset \omega^{\prime} \subset \subset \omega$, that

$$
\left|A_{n}(j) \cap \omega^{\prime \prime}\right| \leq c_{0} \frac{\|\nabla \eta\|_{L^{\infty}(\Omega)}^{2^{*}}\left|A_{n}(k) \cap \omega^{\prime}\right|^{\frac{2^{*}}{2}}}{(j-k)^{2^{*}}} .
$$

Now we consider $R_{0}=\operatorname{dist}\left(\omega^{\prime \prime}, \omega\right)$. Define

$$
\omega_{r}=\left\{x \in \Omega: \operatorname{dist}\left(x, \omega^{\prime \prime}\right)<r\right\}
$$

and

$$
m(k, r)=\left|A_{n}(k) \cap \omega_{r}\right|,
$$


for every $0<r<R_{0}$ and $k>0$. Choosing $0 \leq r<R<R_{0}$ and $\eta$ such that $\|\nabla \eta\|_{L^{\infty}(\Omega)} \leq$ $\frac{c_{1}}{R-r}$ and taking $\omega^{\prime \prime}=\omega_{r}$ and $\omega^{\prime}=\omega_{R}$ in (3.9), we deduce

$$
m(j, r) \leq c_{2} \frac{m(k, R)^{\frac{2^{*}}{2}}}{(j-k)^{2^{*}}(R-r)^{2^{*}}},
$$

where $c_{2}=c_{0} c_{1}^{2^{*}}$. From this inequality, it follows, applying Lemma 3.5, that there exists $M_{\omega^{\prime}}>0$ (independent on $n$ ) such that

$$
\left\|z_{n}^{+}\right\|_{L^{\infty}\left(\omega^{\prime}\right)} \leq M_{\omega^{\prime}} .
$$

Recalling the definition of $z_{n}$ in terms of $u_{n}$, we therefore have

$$
u_{n}=(n+1)^{\frac{1}{n+1}} \mathrm{e}^{-\frac{z n}{n+1}} \geq(n+1)^{\frac{1}{n+1}} \mathrm{e}^{-\frac{M_{\omega^{\prime}}}{n+1}} \text { in } \omega^{\prime},
$$

which is (3.6).

We conclude this section with the following remark:

Remark 3.8 As a consequence of estimates (3.5) and (3.6), we thus have

$$
\lim _{n \rightarrow+\infty} u_{n}=1 \text { uniformly in } \omega^{\prime} .
$$

Repeating this argument for every $\omega^{\prime}$ contained in $\omega$, we have that $u_{n}$ converges to 1 on $\omega$.

\section{Proofs of Theorems 2.2 and 2.3}

We start with the proof of Theorem 2.2, in which we recall that $\omega=\{f>0\}$ is compactly contained in $\Omega$.

Proof of Theorem 2.2 We have already proved that

$$
\left\|u_{n}\right\|_{L^{\infty}(\Omega)} \leq\left(C(n+1)\|f\|_{L^{\infty}(\Omega)}\right)^{\frac{1}{n+1}},
$$

so that $u_{n}$ is bounded in $L^{\infty}(\Omega)$. This implies that there exists $u$ in $L^{\infty}(\Omega)$ such that $u_{n}$ *-weakly converges to $u$ in $L^{\infty}(\Omega)$ and, by Remark 3.8, $u \equiv 1$ in $\omega$. We are now going to prove that the right-hand side of the equation in (2.3) is bounded in $L^{1}(\Omega)$ uniformly in $n$. As a matter of fact, if $u_{n}$ is the solution of (2.3), from Theorem 3.1 and Remark 3.3, it follows that $u_{n} \in W_{0}^{1,2}(\Omega), u_{n} \geq c_{\omega, n}>0$ in $\omega$ and $\frac{f}{u_{n}^{n}}$ belongs to $L^{\infty}(\Omega)$. Then, we have, by the results in [37], that

$$
u_{n}(x)=\int_{\Omega} G(x, y) \frac{f(y)}{u_{n}^{n}(y)} \mathrm{d} y, \quad \forall x \in \Omega,
$$

where $G(x, \cdot)$ is the Green function of the linear differential operator defined by the adjoint matrix $M^{*}(x)$ of $M(x)$, i.e., the unique duality solution of

$$
\begin{cases}-\operatorname{div}\left(M^{*}(x) \nabla G(x, \cdot)\right)=\delta_{x} & \text { in } \Omega, \\ G(x, \cdot)=0 & \text { on } \partial \Omega,\end{cases}
$$


where $\delta_{x}$ is the Dirac delta concentrated at $x$ in $\Omega$. It is well known (see, for example, [31]) that for every $\omega^{\prime} \subset \subset \Omega$ there exists $K>0$ such that

$$
G(x, y) \geq \frac{K}{|x-y|^{N-2}}, \quad \forall x, y \in \omega^{\prime} .
$$

Fix now $\bar{x}$ in $\Omega \backslash \bar{\omega}$, let $\omega^{\prime \prime} \subset \subset \Omega$ be such that $\omega \subset \omega^{\prime \prime}$ and $\bar{x}$ belongs to $\omega^{\prime \prime}$, and let $K$ be such that (4.2) holds. We then have

$$
\begin{aligned}
\left(C(n+1)\|f\|_{L^{\infty}(\Omega)}\right)^{\frac{1}{n+1}} \geq u_{n}(\bar{x}) & =\int_{\Omega} G(\bar{x}, y) \frac{f(y)}{u_{n}^{n}(y)} \mathrm{d} y \\
& \geq \int_{\Omega} \frac{K}{|\bar{x}-y|^{N-2}} \frac{f(y)}{u_{n}^{n}(y)} \mathrm{d} y \\
& \geq \frac{K}{\operatorname{diam}(\Omega)^{N-2}} \int_{\omega} \frac{f(y)}{u_{n}^{n}(y)} \mathrm{d} y .
\end{aligned}
$$

Therefore, there exists $M>0$ such that

$$
\int_{\omega} \frac{f(x)}{u_{n}^{n}}=\int_{\Omega} \frac{f(x)}{u_{n}^{n}} \leq M,
$$

i.e., the right-hand side of the equation in (2.3) is bounded in $L^{1}(\Omega)$. Observe now that for every $\omega^{\prime} \subset \subset \omega$ there exists $M_{\omega^{\prime}}$ such that

$$
u_{n}(x) \geq(n+1)^{\frac{1}{n+1}} \mathrm{e}^{-\frac{M_{\omega^{\prime}}}{n+1}}, \quad \text { in } \omega^{\prime} .
$$

Therefore,

$$
\int_{\omega^{\prime}} \frac{f(x)}{u_{n}^{n}} \leq \frac{\left|\omega^{\prime}\right| \mathrm{e}^{\frac{n M_{\omega^{\prime}}}{n+1}}\|f\|_{L^{\infty}(\Omega)}}{(n+1)^{\frac{n}{n+1}}}
$$

so that

$$
\lim _{n \rightarrow+\infty} \int_{\omega^{\prime}} \frac{f(x)}{u_{n}^{n}}=0,
$$

i.e., the right-hand side converges to zero in $L_{\text {loc }}^{1}(\omega)$. Let now $\mu$ be the bounded Radon measure such that

$$
\frac{f(x)}{u_{n}^{n}} \rightarrow \mu, \quad \text { in the } * \text {-weak topology of measures. }
$$

Clearly, by the assumption on $f, \mu\llcorner(\Omega \backslash \bar{\omega})=0$, and, by (4.4), $\mu\llcorner\omega=0$, so that $\mu=\mu\left\llcorner\partial \omega\right.$. Moreover, by Remark 3.3, we can take $u_{n}$ as test function in (2.3) and we obtain, using (2.1), (4.1) and (4.3), that

$$
\int_{\Omega}\left|\nabla u_{n}\right|^{2} \leq \int_{\Omega} \frac{f(x) u_{n}}{u_{n}^{n}} \leq\left\|u_{n}\right\|_{L^{\infty}(\Omega)} \int_{\Omega} \frac{f(x)}{u_{n}^{n}} \leq C,
$$

then $u_{n}$ weakly converges to $u$ in $W_{0}^{1,2}(\Omega)$ as $n$ tends to infinity. Recalling that, by Remark $3.3, u_{n}$ is the (unique) weak solution of (2.3), that is,

$$
\int_{\Omega} M(x) \nabla u_{n} \cdot \nabla \varphi=\int_{\Omega} \frac{f \varphi}{u_{n}^{n}}, \quad \forall \varphi \in W_{0}^{1,2}(\Omega),
$$


we obtain, letting $n$ tend to infinity, that

$$
\int_{\Omega} M(x) \nabla u \cdot \nabla \varphi=\int_{\Omega} \varphi \mathrm{d} \mu, \quad \forall \varphi \in C_{0}^{1}(\Omega),
$$

so that $u$ is a distributional solution with finite energy of the limit problem (2.4).

Remark 4.1 We observe that $u_{n}$ is also the unique duality solution of (2.3), i.e.,

$$
\int_{\Omega} u_{n} g=\int_{\Omega} \frac{f}{u_{n}^{n}} v, \quad \forall g \in L^{\infty}(\Omega),
$$

where $v \in W_{0}^{1,2}(\Omega) \cap L^{\infty}(\Omega)$ is the unique weak solution of

$$
\left\{\begin{aligned}
-\operatorname{div}\left(M^{*}(x) \nabla v\right) & =g & & \text { in } \Omega, \\
v & =0 & & \text { on } \partial \Omega .
\end{aligned}\right.
$$

This implies, letting $n$ tend to infinity in (4.7) and using the standard results contained in [37], that $u$ is the unique duality solution of (2.4).

Now we prove Theorem 2.3. Here, let us recall that for every $\omega \subset \subset \Omega$ there exists $c_{\omega}>0$ such that $f \geq c_{\omega}$ in $\omega$ and that $\left\{\omega_{n}\right\}$ is an increasing sequence of compactly contained subsets of $\Omega$ such that their union is $\Omega$.

Proof of Theorem 2.3 Let $u_{n}$ be the solution of (2.5). It follows, from the fact that $f(x) \chi_{\omega_{n}}(x)$ has compact support in $\Omega$ and using Remark 3.3, that $u_{n}$ belongs to $W_{0}^{1,2}(\Omega)$ and $\frac{f(x) \chi_{\omega_{n}}(x)}{u_{n}^{n}}$ belongs to $L^{1}(\Omega)$. Once again as a consequence of Theorem 3.2, we have that $\left\{u_{n}\right\}$ is bounded in $L^{\infty}(\Omega)$. Then, there exists $u$ in $L^{\infty}(\Omega)$ such that $u_{n}$ *-weakly converges to $u$ in $L^{\infty}(\Omega)$. Moreover, by Remark 3.8, we deduce that $u_{n}$ uniformly converges to 1 in $\omega$, for every $\omega \subset \subset \Omega$, hence $u \equiv 1$ in $\Omega$. If we assume that the sequence $\left\{\frac{f(x) \chi_{\omega_{n}}(x)}{u_{n}^{n}}\right\}$ is bounded in $L^{1}(\Omega)$, then it *-weakly converges to $\mu$ in the topology of measure. Repeating the same arguments contained in Remark 4.1, we obtain

$$
\int_{\Omega} u g=\int_{\Omega} v d \mu, \quad \forall g \in L^{\infty}(\Omega)
$$

where $v$ in $W_{0}^{1,2}(\Omega)$ is the weak solution of (4.8). Then, $u$ in $L^{\infty}(\Omega)$ is the duality solution of (2.4), so that $u$ belongs to $W_{0}^{1,1}(\Omega)$. Since $u \equiv 1$ in $\Omega$, there is a contradiction. Hence, the right-hand side of (2.5) is not bounded in $L^{1}(\Omega)$ and there cannot be any limit equation.

\section{One-dimensional solutions and Proof of Theorem 2.6}

First we prove a result that makes the link between a distributional solution of (2.3) and a distributional solution with finite energy of (2.6) rigorous.

Proposition 5.1 Let $f$ be a nonnegative function belonging to $L^{\infty}(\Omega)$. If $u_{n}$ is a solution of (2.3) given by Theorem 3.1, then $v_{n}=\frac{u_{n}^{n+1}}{n+1}$ is a distributional solution of (2.6) with finite energy. 
Proof We already know, by Theorem 3.2, that $u_{n}^{n+1}$ belongs to $W_{0}^{1,2}(\Omega)$, so that $v_{n}$ belongs to $W_{0}^{1,2}(\Omega)$. With the same argument, we have that $u_{n}^{n}$ belongs to $W_{0}^{1,2}(\Omega)$. Let $\varphi$ be a function in $C_{c}^{1}(\Omega)$, we have that $u_{n}^{n} \varphi$ is a function in $W_{0}^{1,2}(\Omega)$ with compact support $(\omega=\operatorname{supp}(\varphi))$. Then, we can take $u_{n}^{n} \varphi$ as test function in (3.1) and we obtain that

$$
\int_{\Omega} \nabla u_{n} \cdot \nabla \varphi u_{n}^{n}+n \int_{\Omega} \nabla u_{n} \cdot \nabla u_{n} u_{n}^{n-1} \varphi=\int_{\Omega} f \varphi .
$$

If we rewrite (5.1), using that $u_{n} \geq c_{\omega, n}$ in $\omega$, we have

$$
\int_{\Omega} \nabla\left(\frac{u_{n}^{n+1}}{n+1}\right) \cdot \nabla \varphi+n \int_{\Omega}\left|\nabla u_{n}\right|^{2} \frac{u_{n}^{2 n}}{u_{n}^{n+1}} \varphi=\int_{\Omega} f \varphi .
$$

Hence, by definition of $v_{n}$, we deduce that

$$
\int_{\Omega} \nabla v_{n} \cdot \nabla \varphi+\frac{n}{n+1} \int_{\Omega} \frac{\left|\nabla v_{n}\right|^{2}}{v_{n}} \varphi=\int_{\Omega} f \varphi,
$$

that is, $v_{n}$ is a distributional solution with finite energy of (2.6).

Remark 5.2 We note that for every $\omega \subset \subset \Omega$ we know, by Theorem 3.1, that $u_{n} \geq c_{\omega, n}$ in $\omega$. Then, $v_{n} \geq \frac{c_{\omega, n}^{n+1}}{n+1}$ in $\omega$. Using this property and that $v_{n}$ has finite energy, we can extend the class of test functions for (2.6) from $C_{c}^{1}(\Omega)$ to $W_{0}^{1,2}(\Omega)$ with compact support.

Now we study (2.3) in the one-dimensional case to better understand what happens, if $f$ is strictly positive, to $u_{n}$ and to the related $v_{n}$ by passing to the limit as $n$ tends to infinity.

Fix $n>3$ in $\mathbb{N}$. We consider (2.3) with $\Omega=(-R, R), R>0, M(x) \equiv I$ and $f \equiv 1$ in $(-R, R)$ so that we have

$$
\left\{\begin{array}{l}
-u_{n}^{\prime \prime}=\frac{1}{u_{n}^{n}} \quad \text { in }(-R, R), \\
u_{n}( \pm R)=0 .
\end{array}\right.
$$

In order to study (5.2), we focus on the solutions $y_{n}$ of the following Cauchy problem

$$
\left\{\begin{array}{l}
-y_{n}^{\prime \prime}(t)=\frac{1}{y_{n}^{n}(t)} \text { for } t \geq 0, \\
y_{n}(0)=\alpha_{n} \\
y_{n}^{\prime}(0)=0
\end{array}\right.
$$

where $\alpha_{n}$ is a positive real number that we will choose later. Defining $w_{n}=\frac{y_{n}}{\alpha_{n}}$, we can rewrite (5.3) as

$$
\left\{\begin{array}{l}
-w_{n}^{\prime \prime}(t)=\frac{1}{\alpha_{n}^{n+1} w_{n}^{n}(t)} \text { for } t \geq 0 \\
w_{n}(0)=1 \\
w_{n}^{\prime}(0)=0
\end{array}\right.
$$

Since $\frac{1}{\alpha_{n}^{n+1} s^{n}}$ is Lipschitz continuous near $s=1$, there exists a unique solution $w_{n}$ locally near $t=0$. It is easy, by a classical iteration argument, to extend the definition interval of $w_{n}$ to $\left[0, T_{n}\right.$ ), where $T_{n}<+\infty$ is the first zero of $w_{n}$ (i.e., $w_{n}\left(T_{n}\right)=0$ ) when it occurs, otherwise 
$T_{n}=+\infty$. Hence, $w_{n}$ is concave $\left(w_{n}^{\prime \prime}(t)<0\right)$, decreasing $\left(w_{n}^{\prime}(t)<0\right)$ and $0<w_{n}(t) \leq 1$ for $t \in\left[0, T_{n}\right)$ and it belongs to $C^{\infty}\left(\left(0, T_{n}\right)\right)$.

Now multiplying the equation by $w_{n}^{\prime}(t)$, we have

$$
-\frac{\left[w_{n}^{\prime}(t)^{2}\right]^{\prime}}{2}=\frac{w_{n}^{\prime}(t)}{\alpha_{n}^{n+1} w_{n}^{n}(t)},
$$

hence, integrating on $[0, s]$, with $0<s<T_{n}$, and recalling that $w_{n}^{\prime}(0)=0$, we have

$$
w_{n}^{\prime}(s)^{2}=\frac{2}{(n-1) \alpha_{n}^{n+1}}\left(w_{n}^{1-n}(s)-1\right) .
$$

Since $w_{n}^{\prime}(s)<0$ we deduce

$$
w_{n}^{\prime}(s)=-\sqrt{\frac{2}{(n-1) \alpha_{n}^{n+1}}}\left(w_{n}^{1-n}(s)-1\right)^{\frac{1}{2}},
$$

we can divide (5.5) by $\left(w_{n}^{1-n}(s)-1\right)^{\frac{1}{2}}$ and integrate on $[0, t]$, with $0 \leq t<T_{n}$, to obtain

$$
\int_{0}^{t} \frac{w_{n}^{\prime}(s)}{\left(w_{n}^{1-n}(s)-1\right)^{\frac{1}{2}}} \mathrm{~d} s=-\sqrt{\frac{2}{(n-1) \alpha_{n}^{n+1}}} t .
$$

Setting $r=w_{n}(s)$ in the first integral of (5.6) and recalling that $w_{n}(0)=1$, we have

$$
\int_{w_{n}(t)}^{1} \frac{r^{\frac{n-1}{2}}}{\left(1-r^{n-1}\right)^{\frac{1}{2}}} \mathrm{~d} r=\sqrt{\frac{2}{(n-1) \alpha_{n}^{n+1}}} t .
$$

Once again we can perform the change of variable $h=1-r^{n-1}$ to deduce

$$
\int_{0}^{1-w_{n}^{n-1}(t)} \frac{1}{h^{\frac{1}{2}}(1-h)^{\frac{n-3}{2(n-1)}}} \mathrm{d} h=\sqrt{\frac{2(n-1)}{\alpha_{n}^{n+1}}} t .
$$

Define $I_{n}(t):=\int_{0}^{1-w_{n}^{n-1}(t)} \frac{1}{h^{\frac{1}{2}}(1-h)^{\frac{n-3}{2(n-1)}}} \mathrm{d} h$ for $t \geq 0$, then $I_{n}(0)=0$ and $I_{n}$ is a continuous positive and increasing function in $\left[0, T_{n}\right)$, so that $I_{n}(t) \leq I_{n}\left(T_{n}\right)$. Thanks to the results in [1], we obtain

$$
I_{n}\left(T_{n}\right)=\int_{0}^{1} \frac{1}{h^{\frac{1}{2}}(1-h)^{\frac{n-3}{2(n-1)}}} \mathrm{d} h=\sqrt{\pi} \frac{\Gamma\left(\frac{1}{2}+\frac{1}{n-1}\right)}{\Gamma\left(\frac{n}{n-1}\right)},
$$

where $\Gamma(s)$ is defined in (1.5). Thus, we can extend $I_{n}(t)$ in $\left[0, T_{n}\right]$ and it is uniformly bounded for every $n \in \mathbb{N}$ and $t \in\left[0, T_{n}\right]$. Moreover, from (5.8) and computing (5.7) for $t=T_{n}$, we have

$$
T_{n}=\sqrt{\frac{\pi \alpha_{n}^{n+1}}{2(n-1)}} \frac{\Gamma\left(\frac{1}{2}+\frac{1}{n-1}\right)}{\Gamma\left(\frac{n}{n-1}\right)} .
$$

We observe that $T_{n}$ and $\alpha_{n}$ are such that if $\alpha_{n}$ tends to infinity also $T_{n}$ tends to infinity. Recalling that we want a solution for (5.2) that is zero if $t=R$, imposing $T_{n}=R$ for every $n$ in $\mathbb{N}$ we find that 


$$
\alpha_{n}=\left(\frac{2 R^{2}(n-1) \Gamma^{2}\left(\frac{n}{n-1}\right)}{\pi \Gamma^{2}\left(\frac{1}{2}+\frac{1}{n-1}\right)}\right)^{\frac{1}{n+1}} .
$$

Hence, with this value of $\alpha_{n}, w_{n}(R)=0$ for every $n$ in $\mathbb{N}$ and $w_{n}$ belongs to $C^{2}((0, R))$. Thanks to the initial condition $w_{n}^{\prime}(0)=0$, we can extend $w_{n}$ to an even function $\tilde{w}_{n}$ on $[-R, R]$ in the following way

$$
\tilde{w}_{n}(t)= \begin{cases}w_{n}(t) & \text { for } t \in[0, R], \\ w_{n}(-t) & \text { for } t \in[-R, 0) .\end{cases}
$$

So $\tilde{w}_{n}$ belongs to $C_{0}^{2}((-R, R))$ and is the classical solution of

$$
\left\{\begin{array}{l}
-\tilde{w}_{n}^{\prime \prime}(t)=\frac{1}{\alpha_{n}^{n+1} \tilde{w}_{n}^{n}(t)} \text { for } t \geq 0 \\
\tilde{w}_{n}( \pm R)=0
\end{array}\right.
$$

Setting $u_{n}(t)=\alpha_{n} \tilde{w}_{n}(t)$ for $t$ in $[-R, R]$ we have that $u_{n}$ belongs to $C_{0}^{2}((-R, R))$ and is the classical solution of (5.2). This implies that $v_{n}(t)=\frac{u_{n}(t)^{n+1}}{n+1}$ is a classical solution (in $\left.C_{0}^{2}((-R, R))\right)$ of

$$
\left\{\begin{array}{l}
-v_{n}^{\prime \prime}+\frac{n}{n+1} \frac{\left|v_{n}^{\prime}\right|^{2}}{v_{n}}=1 \text { in }(-R, R) \\
v_{n}( \pm R)=0
\end{array}\right.
$$

that is, (2.6) in the one-dimensional case. Multiplying Eq. (5.12) by $v_{n}$ and integrating by parts on $(-R, R)$, we obtain that $\left\{v_{n}\right\}$ is bounded in $W_{0}^{1,2}((-R, R))$. By definition of $v_{n}$, this implies that $\left\{\tilde{w}_{n}^{n+1}\right\}$ is bounded in $W_{0}^{1,2}((-R, R))$. Using the Rellich-Kondrachov theorem, we deduce that there exist a subsequence, still indexed by $\tilde{w}_{n}^{n+1}$, and a function $g:(-R, R) \rightarrow[0,1]$ in $C_{0}((-R, R))$ such that $\tilde{w}_{n}^{n+1}$ uniformly converges to $g$ in $(-R, R)$. We want to make $g$ explicit.

By definition of $\tilde{w}_{n}$, it follows that

$$
\lim _{n \rightarrow \infty} w_{n}^{n-1}(t)=\lim _{n \rightarrow \infty}\left(w_{n}^{n+1}(t)\right)^{\frac{n-1}{n+1}}=g(t),
$$

uniformly in $(0, R)$. Combining (5.7) and (5.10), we obtain

$$
\int_{0}^{1-w_{n}^{n-1}(t)} \frac{1}{h^{\frac{1}{2}}(1-h)^{\frac{n-3}{2(n-1)}}} \mathrm{d} h=\frac{\sqrt{\pi}}{R} \frac{\Gamma\left(\frac{1}{2}+\frac{1}{n-1}\right)}{\Gamma\left(\frac{n}{n-1}\right)} t .
$$

Passing to the limit in (5.13) as $n$ tends to infinity, we obtain the explicit expression of $g$. Indeed, we have, by Lebesgue theorem and from well-known result of integral calculus, that

$$
\begin{aligned}
2 \arcsin (\sqrt{1-g(t)}) & =\lim _{n \rightarrow \infty} \int_{0}^{1-w_{n}^{n-1}(t)} \frac{1}{h^{\frac{1}{2}}(1-h)^{\frac{n-3}{2(n-1)}}} \mathrm{d} h \\
& =\lim _{n \rightarrow \infty} \frac{\sqrt{\pi}}{R} \frac{\Gamma\left(\frac{1}{2}+\frac{1}{n-1}\right)}{\Gamma\left(\frac{n}{n-1}\right)} t=\frac{\pi}{R} t .
\end{aligned}
$$


It follows that

$$
g(t)=1-\sin ^{2}\left(\frac{\pi}{2 R} t\right)=\cos ^{2}\left(\frac{\pi}{2 R} t\right) .
$$

So $g$ is an even $C^{\infty}$ function defined on $\mathbb{R}$, in particular on $[-R, R]$.

Fix now $t$ in $(-R, R)$. We want to prove that $\tilde{w}_{n}(t)$ tends to 1 as $n$ tends to infinity. We assume, by contradiction, that

$$
\lim _{n \rightarrow \infty} \tilde{w}_{n}(t)=\beta<1
$$

Defining $\varepsilon:=\frac{1-\beta}{2}$, we deduce, for $n$ large enough, that $\tilde{w}_{n}(t) \leq 1-\varepsilon$ so that

$$
\tilde{w}_{n}^{n+1}(t) \leq(1-\varepsilon)^{n+1},
$$

and, letting $n$ tend to infinity, we obtain $\cos ^{2}\left(\frac{\pi}{2 R} t\right)=0$. Since $t \neq \pm R$, we find a contradiction; then $\tilde{w}_{n}(t)$ tends to 1 , as $n$ tends to infinity, for every $t$ in $(-R, R)$.

Now we return to problem (5.2) recalling that $u_{n}(t)=\alpha_{n} \tilde{w}_{n}(t)$. From (5.10) and using that $\tilde{w}_{n}(t)$ tends to 1 , as $n$ tends to infinity, for $t$ in $(-R, R)$, it follows that

$$
\lim _{n \rightarrow \infty} u_{n}(t)=1, \quad \forall t \in(-R, R) .
$$

This result is exactly the one-dimensional version of Remark 3.8. From (5.10), we deduce that

$$
v_{n}(t)=\frac{2 R^{2}(n-1) \Gamma^{2}\left(\frac{n}{n-1}\right)}{\pi(n+1) \Gamma^{2}\left(\frac{1}{2}+\frac{1}{n-1}\right)} \tilde{w}_{n}^{n+1}(t),
$$

so that we have that there exists a limit function $v:[-R, R] \rightarrow \mathbb{R}$ such that

$$
v(t)=\lim _{n \rightarrow \infty} v_{n}(t)=\frac{2 R^{2}}{\pi^{2}} \cos ^{2}\left(\frac{\pi}{2 R} t\right) .
$$

After a little algebra, we obtain that $v$ is a classical solution of

$$
\left\{\begin{array}{l}
-v^{\prime \prime}+\frac{\left|v^{\prime}\right|^{2}}{v}=1 \text { in }(-R, R), \\
v_{n}( \pm R)=0
\end{array}\right.
$$

that is, (2.8). Thus, we have proved Theorem 2.6 in the one-dimensional case.

Finally, we prove Theorem 2.6 in the $N$-dimensional case; here, we recall that $f$ is strictly positive.

Proof of Theorem 2.6 Let $u_{n}$ be the solution of (2.3) given by Theorem 3.1. It follows from Proposition 5.1 that $v_{n}$ are distributional solutions of (2.6).

By assumption for every $\omega \subset \subset \Omega$, there exists a positive constant $c_{\omega}$ such that $f \geq c_{\omega}$. This implies, by Theorem 3.7, that

$$
u_{n} \geq(n+1)^{\frac{1}{n+1}} \mathrm{e}^{-\frac{M_{\omega}}{n+1}},
$$

then

$$
v_{n} \geq \mathrm{e}^{-M_{\omega}}, \quad \forall \omega \subset \subset \Omega,
$$


with $M_{\omega}$ a positive constant depending only on $\omega$. So $v_{n}$ is locally uniformly positive. Moreover, by Theorem 3.2, we have that $v_{n}$ belongs to $W_{0}^{1,2}(\Omega)$ and

$$
\left\|v_{n}\right\|_{L^{\infty}(\Omega)} \leq C\|f\|_{L^{\infty}(\Omega)},
$$

where $C$ is a positive constant.

Choosing a nonnegative $\varphi$ belonging to $C_{c}^{1}(\Omega)$ as test function in (2.6) and dropping the nonnegative integral involving the quadratic gradient term, we deduce that

$$
\int_{\Omega} \nabla v_{n} \cdot \nabla \varphi \leq \int_{\Omega} f \varphi .
$$

As a consequence of the density of $C_{c}^{1}(\Omega)$ in $W_{0}^{1,2}(\Omega)$, we can extend (5.15) for every nonnegative $\varphi$ in $W_{0}^{1,2}(\Omega)$. Choosing $v_{n}$ as test function and using Hölder's inequality and the Sobolev embedding theorem, we obtain

$$
\int_{\Omega}\left|\nabla v_{n}\right|^{2} \leq \int_{\Omega} f v_{n} \leq\|f\|_{L^{\frac{2 N}{N+2}}(\Omega)}\left\|v_{n}\right\|_{L^{2^{*}}(\Omega)} \leq \mathcal{S}\|f\|_{L^{\frac{2 N}{N+2}}(\Omega)}\left\|v_{n}\right\|_{W_{0}^{1,2}(\Omega)},
$$

where $\mathcal{S}$ is the Sobolev constant. Hence, $\left\{v_{n}\right\}$ is bounded in $W_{0}^{1,2}(\Omega)$. Thus, up to a subsequence, it follows that there exists $v$ belonging to $W_{0}^{1,2}(\Omega) \cap L^{\infty}(\Omega)$ such that

$$
\begin{aligned}
& v_{n} \rightarrow v \text { weakly in } W_{0}^{1,2}(\Omega) \text { and weakly-* in } L^{\infty}(\Omega), \\
& v_{n} \rightarrow v \text { strongly in } L^{q}(\Omega), \forall q<+\infty, \text { and a.e. in } \Omega .
\end{aligned}
$$

In order to pass to the limit in (2.6), we first prove that $v_{n}$ strongly converges to $v$ in $W_{\text {loc }}^{1,2}(\Omega)$, that is

$$
\lim _{n \rightarrow+\infty} \int_{\Omega}\left|\nabla\left(v_{n}-v\right)\right|^{2} \varphi=0, \quad \forall \varphi \in C_{c}^{1}(\Omega) \text { with } \varphi \geq 0 .
$$

We consider the function $\phi_{\lambda}(s)$ defined in (1.6), and choosing $\phi_{\lambda}\left(v_{n}-v\right) \varphi$ as test function in (2.6), we obtain

$$
\begin{gathered}
\int_{\Omega} \nabla v_{n} \cdot \nabla\left(v_{n}-v\right) \phi_{\lambda}^{\prime}\left(v_{n}-v\right) \varphi+\int_{\Omega} \nabla v_{n} \cdot \nabla \varphi \phi_{\lambda}\left(v_{n}-v\right) \\
+\frac{n}{n+1} \int_{\Omega} \frac{\left|\nabla v_{n}\right|^{2}}{v_{n}} \phi_{\lambda}\left(v_{n}-v\right) \varphi=\int_{\Omega} f \phi_{\lambda}\left(v_{n}-v\right) \varphi .
\end{gathered}
$$

It follows from (5.16) and using Lebesgue theorem that

$$
\lim _{n \rightarrow+\infty} \int_{\Omega} \nabla v_{n} \cdot \nabla \varphi \phi_{\lambda}\left(v_{n}-v\right)=0 \text { and } \lim _{n \rightarrow+\infty} \int_{\Omega} f \phi_{\lambda}\left(v_{n}-v\right) \varphi=0 .
$$

Thus,

$$
\int_{\Omega} \nabla v_{n} \cdot \nabla\left(v_{n}-v\right) \phi_{\lambda}^{\prime}\left(v_{n}-v\right) \varphi+\frac{n}{n+1} \int_{\Omega} \frac{\left|\nabla v_{n}\right|^{2}}{v_{n}} \phi_{\lambda}\left(v_{n}-v\right) \varphi=\epsilon(n) .
$$

Moreover, setting $\omega_{\varphi}=\operatorname{supp}(\varphi)$ and using (5.14), we deduce that

$$
\begin{aligned}
\frac{n}{n+1} \int_{\Omega} \frac{\left|\nabla v_{n}\right|^{2}}{v_{n}} \phi_{\lambda}\left(v_{n}-v\right) \varphi & \geq-\frac{n}{n+1} \int_{\Omega} \frac{\left|\nabla v_{n}\right|^{2}}{v_{n}}\left|\phi_{\lambda}\left(v_{n}-v\right)\right| \varphi \\
& \geq-\mathrm{e}^{M_{\omega \varphi}} \int_{\Omega}\left|\nabla v_{n}\right|^{2}\left|\phi_{\lambda}\left(v_{n}-v\right)\right| \varphi,
\end{aligned}
$$


so that

$$
\int_{\Omega} \nabla v_{n} \cdot \nabla\left(v_{n}-v\right) \phi_{\lambda}^{\prime}\left(v_{n}-v\right) \varphi-\mathrm{e}^{M_{\omega_{\varphi}}} \int_{\Omega}\left|\nabla v_{n}\right|^{2}\left|\phi_{\lambda}\left(v_{n}-v\right)\right| \varphi=\epsilon(n) .
$$

We can add the following term to (5.19)

$$
-\int_{\Omega} \nabla v \cdot \nabla\left(v_{n}-v\right) \phi_{\lambda}^{\prime}\left(v_{n}-v\right) \varphi
$$

and, noting that by (5.16) this quantity tends to 0 letting $n$ go to infinity, we obtain

$$
\int_{\Omega}\left|\nabla\left(v_{n}-v\right)\right|^{2} \phi_{\lambda}^{\prime}\left(v_{n}-v\right) \varphi-\mathrm{e}^{M_{\omega \varphi}} \int_{\Omega}\left|\nabla v_{n}\right|^{2}\left|\phi_{\lambda}\left(v_{n}-v\right)\right| \varphi=\epsilon(n) .
$$

Since using once again (5.16) we have

$$
\begin{aligned}
& \int_{\Omega}\left|\nabla v_{n}\right|^{2}\left|\phi_{\lambda}\left(v_{n}-v\right)\right| \varphi \leq 2 \int_{\Omega}\left|\nabla\left(v_{n}-v\right)\right|^{2}\left|\phi_{\lambda}\left(v_{n}-v\right)\right| \varphi \\
& \quad+2 \int_{\Omega}|\nabla v|^{2}\left|\phi_{\lambda}\left(v_{n}-v\right)\right| \varphi=2 \int_{\Omega}\left|\nabla\left(v_{n}-v\right)\right|^{2}\left|\phi_{\lambda}\left(v_{n}-v\right)\right| \varphi+\epsilon(n),
\end{aligned}
$$

we deduce that

$$
\int_{\Omega}\left|\nabla\left(v_{n}-v\right)\right|^{2}\left\{\phi_{\lambda}^{\prime}\left(v_{n}-v\right)-2 \mathrm{e}^{M_{\omega \varphi}}\left|\phi_{\lambda}\left(v_{n}-v\right)\right|\right\} \varphi=\epsilon(n) .
$$

Choosing $\lambda \geq \mathrm{e}^{2 M_{\omega_{\varphi}}}$, thanks to (1.7) we have that $\left\{\phi_{\lambda}^{\prime}\left(v_{n}-v\right)-2 \mathrm{e}^{M_{\omega_{\varphi}}}\left|\phi_{\lambda}\left(v_{n}-v\right)\right|\right\} \geq \frac{1}{2}$, hence (5.17) holds and

$$
v_{n} \rightarrow v \text { strongly in } W_{\mathrm{loc}}^{1,2}(\Omega) .
$$

Now we pass to the limit in (2.6) with test functions $\varphi$ belonging to $W_{0}^{1,2}(\Omega) \cap L^{\infty}(\Omega)$ with compact support. We have, by (5.17), that

$$
\lim _{n \rightarrow+\infty} \int_{\Omega} \nabla v_{n} \cdot \nabla \phi=\int_{\Omega} \nabla v \cdot \nabla \varphi
$$

and, using (5.21), (5.14) with $\omega=\operatorname{supp}(\varphi)$ and Lebesgue theorem, we deduce

$$
\lim _{n \rightarrow+\infty} \frac{n}{n+1} \int_{\Omega} \frac{\left|\nabla v_{n}\right|^{2}}{v_{n}} \varphi=\int_{\Omega} \frac{|\nabla v|^{2}}{v} \varphi,
$$

so that

$$
\int_{\Omega} \nabla v \cdot \nabla \varphi+\int_{\Omega} \frac{|\nabla v|^{2}}{v} \varphi=\int_{\Omega} f \varphi,
$$

for all $\varphi$ in $W_{0}^{1,2}(\Omega) \cap L^{\infty}(\Omega)$ with compact support.

Let $\varphi$ be a nonnegative function in $W_{0}^{1,2}(\Omega) \cap L^{\infty}(\Omega)$. Let $\left\{\varphi_{m}\right\}$ in $C_{c}^{1}(\Omega)$ be a sequence of nonnegative functions that converges to $\varphi$ strongly in $W_{0}^{1,2}(\Omega)$. Taking $\varphi_{m} \wedge \varphi$, which belongs to $W_{0}^{1,2}(\Omega) \cap L^{\infty}(\Omega)$ with compact support, as test function in (5.22), we obtain

$$
\int_{\Omega} \frac{|\nabla v|^{2}}{v}\left(\varphi_{m} \wedge \varphi\right)=\int_{\Omega} f\left(\varphi_{m} \wedge \varphi\right)-\int_{\Omega} \nabla v \cdot \nabla\left(\varphi_{m} \wedge \varphi\right) .
$$


Since $\varphi_{m} \wedge \varphi$ strongly converges to $\varphi$ in $W_{0}^{1,2}(\Omega)$, we have

$$
\lim _{m \rightarrow+\infty} \int_{\Omega}\left\{f\left(\varphi_{m} \wedge \varphi\right)-\int_{\Omega} \nabla v \cdot \nabla\left(\varphi_{m} \wedge \varphi\right)\right\}=\int_{\Omega} f \varphi-\int_{\Omega} \nabla v \cdot \nabla \varphi .
$$

Moreover, $\frac{|\nabla v|^{2}}{v}\left(\varphi_{m} \wedge \varphi\right)$ is a nonnegative function that converges to $\frac{|\nabla v|^{2}}{v} \varphi$ almost everywhere in $\Omega$. Applying Fatou's lemma on the left-hand side of (5.23) and using (5.24), we deduce that

$$
\int_{\Omega} \frac{|\nabla v|^{2}}{v} \varphi \leq \liminf _{m \rightarrow+\infty} \int_{\Omega} \frac{|\nabla v|^{2}}{v}\left(\varphi_{m} \wedge \varphi\right)=\int_{\Omega} f \varphi-\int_{\Omega} \nabla v \cdot \nabla \varphi,
$$

so that $\frac{|\nabla v|^{2}}{v} \varphi$ belongs to $L^{1}(\Omega)$. Since $\frac{|\nabla v|^{2}}{v}\left(\varphi_{m} \wedge \varphi\right) \leq \frac{|\nabla v|^{2}}{v} \varphi$, by Lebesgue theorem, we have

$$
\lim _{m \rightarrow+\infty} \int_{\Omega} \frac{|\nabla v|^{2}}{v}\left(\varphi_{m} \wedge \varphi\right)=\int_{\Omega} \frac{|\nabla v|^{2}}{v} \varphi .
$$

As a consequence of (5.24) and (5.25), we obtain

$$
\int_{\Omega} \nabla v \cdot \nabla \varphi+\int_{\Omega} \frac{|\nabla v|^{2}}{v} \varphi=\int_{\Omega} f \varphi, \quad \forall \varphi \geq 0 \text { in } W_{0}^{1,2}(\Omega) \cap L^{\infty}(\Omega) .
$$

Furthermore, taking $\frac{T_{\varepsilon}(v)}{\varepsilon}$ as test function in (5.26) and dropping a positive term, we deduce

$$
\int_{\Omega} \frac{|\nabla v|^{2}}{v} \frac{T_{\varepsilon}(v)}{\varepsilon} \leq \int_{\Omega} f \frac{T_{\varepsilon}(v)}{\varepsilon} .
$$

Applying Fatou's lemma on the left-hand side of (5.27) and noting that $T_{\varepsilon}(v) \leq \varepsilon$, we have

$$
\int_{\Omega} \frac{|\nabla v|^{2}}{v} \leq \liminf _{\varepsilon \rightarrow 0} \int_{\Omega} \frac{|\nabla v|^{2}}{v} \frac{T_{\varepsilon}(v)}{\varepsilon} \leq \int_{\Omega} f,
$$

so $\frac{|\nabla v|^{2}}{v}$ belongs to $L^{1}(\Omega)$. Since we can write each $\varphi \in W_{0}^{1,2}(\Omega) \cap L^{\infty}(\Omega)$ as the difference between its positive and its negative part, we trivially deduce that (5.26) holds for all $\varphi \in$ $W_{0}^{1,2}(\Omega) \cap L^{\infty}(\Omega)$, so that $v$ is a weak solution of (2.8).

Remark 5.3 We note that we can also consider test functions only belonging to $W_{0}^{1,2}(\Omega)$ in (5.26). Indeed, let $\varphi$ be in $W_{0}^{1,2}(\Omega)$, then $T_{k}\left(\varphi^{+}\right)$is a positive function belonging to $W_{0}^{1,2}(\Omega) \cap L^{\infty}(\Omega)$ that strongly converges to $\varphi^{+}$in $W_{0}^{1,2}(\Omega)$ as $k$ tends to infinity. Taking $T_{k}\left(\varphi^{+}\right)$as test function in (5.26) and letting $k$ tend to infinity, by Lebesgue theorem and Beppo Levi theorem, we deduce

$$
\int_{\Omega} \nabla v \cdot \nabla \varphi^{+}+\int_{\Omega} \frac{|\nabla v|^{2}}{v} \varphi^{+}=\int_{\Omega} f \varphi^{+} .
$$

In the same way, we obtain

$$
\int_{\Omega} \nabla v \cdot \nabla \varphi^{-}+\int_{\Omega} \frac{|\nabla v|^{2}}{v} \varphi^{-}=\int_{\Omega} f \varphi^{-},
$$

so that subtracting (5.29) to (5.28) we have that (5.26) holds for every $\varphi$ belonging to $W_{0}^{1,2}(\Omega)$. 
Remark 5.4 To prove that $\left\{v_{n}\right\}$ is bounded in $W_{0}^{1,2}(\Omega)$ and (5.16), we only used that $f$ is nonnegative and belongs to $L^{\infty}(\Omega)$.

\section{Proof of Theorem 2.7}

Here, we prove Theorem 2.7. We fix $n>3$ in $\mathbb{N}$.

Proof of Theorem 2.7 First, we study the behavior of the weak solution of (2.9) given by Theorem 3.1. In order to study $u_{n}$, we use the construction of one-dimensional solutions done in the previous section, in which we have proved that there exists a function $w_{n}$ in $C^{2}\left(\left(0, T_{n}\right)\right)$ classical solution of

$$
\left\{\begin{array}{l}
-w_{n}^{\prime \prime}(t)=\frac{1}{\alpha_{n}^{n+1} w_{n}^{n}(t)} \quad \text { in }\left(0, T_{n}\right), \\
w_{n}(0)=1 \\
w_{n}^{\prime}(0)=0
\end{array}\right.
$$

where $T_{n}$ is the first zero of $w_{n}$. We recall that $0<w_{n}(t)<1, w_{n}$ is concave $\left(w_{n}^{\prime \prime}(t)<0\right)$ and decreasing $\left(w_{n}^{\prime}(t)<0\right)$ for every $t$ in $\left(0, T_{n}\right)$. Moreover, we have obtained that

$$
w_{n}^{\prime}(t)=-\sqrt{\frac{2}{(n-1) \alpha_{n}^{n+1}}}\left(w_{n}^{1-n}(t)-1\right)^{\frac{1}{2}},
$$

and, by integrating, that

$$
S_{n}\left(1-w_{n}^{n-1}(t)\right):=\int_{0}^{1-w_{n}^{n-1}(t)} \frac{1}{h^{\frac{1}{2}}(1-h)^{\frac{n-3}{2(n-1)}}} \mathrm{d} h=\sqrt{\frac{2(n-1)}{\alpha_{n}^{n+1}}} t,
$$

for every $t$ in $\left[0, T_{n}\right)$ so that $S_{n}:[0,1) \rightarrow\left[0, S_{n}(1)\right)$ is a nonnegative, continuous and strictly increasing function. Recalling (5.8), we have that $S_{n}(1)=I_{n}\left(T_{n}\right)$, that is uniformly bounded, and thus, we can extend $S_{n}$ in 1 to have $S_{n}:[0,1] \rightarrow\left[0, S_{n}(1)\right]$. Then, there exists the inverse function $S_{n}^{-1}:\left[0, S_{n}(1)\right] \rightarrow[0,1]$. Furthermore, we recall that

$$
T_{n}=\sqrt{\frac{\pi \alpha_{n}^{n+1}}{2(n-1)}} \frac{\Gamma\left(\frac{1}{2}+\frac{1}{n-1}\right)}{\Gamma\left(\frac{n}{n-1}\right)} .
$$

In order to have $1<T_{n}<+\infty$ for every $n$ we can choose $\alpha_{n}=\left(c_{n}(n-1)\right)^{\frac{1}{n+1}}$, with $c_{n}$ a positive constant such that

$$
c_{n}>\frac{2 \Gamma^{2}\left(\frac{n}{n-1}\right)}{\pi \Gamma^{2}\left(\frac{1}{2}+\frac{1}{n-1}\right)}=: \underline{c}_{n}, \quad \forall n \text { in } \mathbb{N} .
$$

Now we consider the following Cauchy problem

$$
\left\{\begin{array}{l}
-y_{n}^{\prime \prime}(t)=\frac{\chi_{(0,1)}}{c_{n}(n-1) y_{n}^{n}(t)} \quad \text { for } t \geq 0, \\
y_{n}(0)=1 \\
y_{n}^{\prime}(0)=0
\end{array}\right.
$$


For every $t$ in $(0,1)$, we have that (6.1) and (6.6) are the same problem, so that there exists $y_{n}(t) \equiv w_{n}(t)$ classical solution of $(6.6)$ in $(0,1)$. Since $y_{n}^{\prime \prime}(t)=0$ for every $t \geq 1$, we deduce that $y_{n}(t)=y_{n}(1)+y_{n}^{\prime}(1)(t-1)=w_{n}(1)+w_{n}^{\prime}(1)(t-1)$ in [1, 2). It follows from (6.2) and by the definition of $\alpha_{n}$ that

$$
w_{n}^{\prime}(1)=-\sqrt{\frac{2}{c_{n}(n-1)^{2}}}\left(w_{n}^{1-n}(1)-1\right)^{\frac{1}{2}} .
$$

Since we want that $y_{n}(2)=0$ for every $n$ in $\mathbb{N}$, we look for $c_{n}$ such that $w_{n}^{\prime}(1)=-w_{n}(1)$. With a little algebra, it follows from (6.7) and (6.3) that is possible if and only if, for every fixed $n$, we have

$$
w_{n}^{n+1}(1)=\frac{2}{c_{n}(n-1)^{2}}\left(1-w_{n}^{n-1}(1)\right)=\frac{2}{c_{n}(n-1)^{2}} S_{n}^{-1}\left(\sqrt{\frac{2}{c_{n}}}\right) .
$$

By Lemma 6.2, there exists a sequence $\left\{c_{n}\right\}$ such that (6.8) holds for every $n$; hence, we have that $y_{n}$ belonging to $C^{1}((0,2))$ is such that

$$
y_{n}(t) \equiv w_{n}(t) \text { in }[0,1], \quad y_{n}(t)=w_{n}(1)(2-t) \text { in }(1,2], \quad y_{n}^{\prime}(0)=y_{n}(2)=0 .
$$

We want that $w_{n}(t) \leq y_{n}(t)$ in $\left[0, T_{n}\right]$. This is true if and only if $T_{n} \leq 2$. If, by contradiction, $T_{n}>2$, we have $w_{n}(t) \equiv y_{n}(t)$ in $[0,1]$ and $-y_{n}^{\prime \prime}(t)<-w_{n}^{\prime \prime}(t)$ in $(1,2]$, so that, by $w_{n}^{\prime}(1)=$ $y_{n}^{\prime}(1)$, we deduce $w_{n}(t)<y_{n}(t)$ in $(1,2]$. It follows from $y_{n}(2)=0$ that $0<w_{n}(2)<0$, which is a contradiction. Then, we obtain $T_{n} \leq 2, w_{n}(t) \leq y_{n}(t)$ in $\left[0, T_{n}\right]$ and, by (6.4), that

$$
c_{n} \leq \frac{8 \Gamma^{2}\left(\frac{n}{n-1}\right)}{\pi \Gamma^{2}\left(\frac{1}{2}+\frac{1}{n-1}\right)}=: \bar{c}_{n}, \quad \forall n \text { in } \mathbb{N} .
$$

Thus, $\left\{c_{n}\right\}$ is bounded and, up to subsequences, there exists a positive real number $c_{\infty}$ such that

$$
\frac{2}{\pi^{2}}=\lim _{n \rightarrow+\infty} \underline{c}_{n} \leq c_{\infty}:=\lim _{n \rightarrow+\infty} c_{n} \leq \lim _{n \rightarrow+\infty} \bar{c}_{n}=\frac{8}{\pi^{2}}
$$

and, respectively,

$$
1 \leq T_{\infty}:=\lim _{n \rightarrow+\infty} T_{n}=\pi \sqrt{\frac{c_{\infty}}{2}} \leq 2 .
$$

As shown in the previous section, it follows from (6.3) that

$$
\lim _{n \rightarrow+\infty} w_{n}^{n+1}(t)=\cos ^{2}\left(\frac{\pi}{2 T_{\infty}} t\right) \text { and } \lim _{n \rightarrow+\infty} w_{n}(t)=1, \quad \text { for } t \in\left(0, T_{\infty}\right) .
$$

Now we suppose that $T_{\infty}>1$. Fix $\beta=\frac{T_{\infty}-1}{2}>0$, so that $1+\beta<T_{\infty}$. We know that for $n$ large enough

$$
w_{n}(1+\beta) \leq y_{n}(1+\beta)=w_{n}(1)(1-\beta) .
$$

By passing to the limit, as $n$ tends to infinity and using (6.11), we obtain $1 \leq 1-\beta$, that is, $\beta \leq 0$. This is a contradiction, then $T_{\infty}=1$ and, therefore, $c_{\infty}=\frac{2}{\pi^{2}}$. 
Recalling that $y_{n}(t) \equiv w_{n}(t)$ in $(0,1)$ and using, once again, (6.11) we have

$$
\lim _{n \rightarrow+\infty} y_{n}^{n+1}(t)=\cos ^{2}\left(\frac{\pi}{2} t\right) \text { and } \lim _{n \rightarrow+\infty} y_{n}(t)=1, \quad \text { for } t \in(0,1) .
$$

It follows from (6.8) and using that $y_{n}(1)=w_{n}(1)$ for every $n$ that

$$
\lim _{n \rightarrow+\infty} y_{n}^{n+1}(1)=0 \text { and } \lim _{n \rightarrow+\infty} y_{n}(1)=1,
$$

hence, by (6.9), we obtain that $y_{n}^{n+1}(t)=w_{n}(1)^{n+1}(2-t)^{n+1}$ and that

$$
\lim _{n \rightarrow+\infty} y_{n}^{n+1}(t)=0 \text { and } \lim _{n \rightarrow+\infty} y_{n}(t)=(2-t), \quad \text { for } t \in(1,2] .
$$

Therefore, by the initial condition $y_{n}^{\prime}(0)=0$, we can extend $y_{n}$ to an even function defined in $(-2,2)$ as follows

$$
\tilde{y}_{n}(t)= \begin{cases}\left(c_{n}(n-1)\right)^{\frac{1}{n+1}} y_{n}(t) & \text { for } t \in[0,2], \\ \left(c_{n}(n-1)\right)^{\frac{1}{n+1}} y_{n}(-t) & \text { for } t \in[-2,0),\end{cases}
$$

so that $\tilde{y}$ belonging to $C_{0}^{1}((-2,2))$ is a weak solution of (2.9). By Remark 3.4, there is a unique weak solution of (2.9), hence $\tilde{y}_{n}(t) \equiv u_{n}(t)$ for every $t$ in $(-2,2)$ and $n$ in $\mathbb{N}$.

Moreover, by Proposition 5.1, setting $v_{n}(t)=\frac{u_{n}^{n+1}(t)}{n+1}$, we have that $v_{n}$ in $C_{0}^{1}((-2,2))$ is a weak solution of (2.10) and, by Remark 5.4, that there exists a function $v$ such that $v_{n}$ weakly converges to $v$ in $W_{0}^{1,2}((-2,2))$ and almost everywhere in $(-2,2)$. As a consequence of (6.12), (6.13) and (6.14), we deduce that

$$
v(t)= \begin{cases}\frac{2}{\pi^{2}} \cos ^{2}\left(\frac{\pi}{2} t\right) & \text { for } t \in(-1,1), \\ 0 & \text { for } t \in[-2,-1] \cup[1,2],\end{cases}
$$

so that $v$ belongs to $C_{0}^{1}(-2,2) \cap C_{0}^{\infty}(-1,1)$. Furthermore, with a little algebra, it follows that $v$ is a classical solution of (2.11).

Remark 6.1 From the proof of Theorem 2.7, we deduce that $u_{n}$ pointwise converges to $u$ defined as follows

$$
u(t)= \begin{cases}(2-t) & \text { for } t \in[1,2], \\ 1 & \text { for } t \in(-1,1), \\ (2+t) & \text { for } t \in[-2,-1]\end{cases}
$$

Moreover, by Theorem 2.2, $u_{n}$ weakly converges to $u$ in $W_{0}^{1,2}((-2,2))$. Hence, we have that

$$
u^{\prime}(t)= \begin{cases}-1 & \text { for } t \in(1,2) \\ 0 & \text { for } t \in(-1,1) \\ 1 & \text { for } t \in(-2,-1)\end{cases}
$$

and $u$ is a distributional solution of

$$
\left\{\begin{array}{c}
-u^{\prime \prime}=-\delta_{-1}+\delta_{1} \text { in }(-2,2), \\
u( \pm 2)=0,
\end{array}\right.
$$

so that we have completely recovered the results of Theorem 2.2. 
To be complete, we show the technical lemma that we needed to prove the theorem.

Lemma 6.2 Letc belong to $\left(c_{0},+\infty\right)$, with $c_{0}=\frac{2 \Gamma^{2}\left(\frac{n}{n-1}\right)}{\pi \Gamma^{2}\left(\frac{1}{2}+\frac{1}{n-1}\right)}$. Let $w_{c}(t)$ be the classical solution of

$$
\left\{\begin{array}{l}
-w_{c}^{\prime \prime}(t)=\frac{1}{c(n-1) w_{c}^{n}(t)} \quad \text { for } t \geq 0 \\
w_{c}(0)=1 \\
w_{c}^{\prime}(0)=0
\end{array}\right.
$$

Let $T_{c}$ be the first zero of $w_{c}$. Then, there exists a unique $\tilde{c}$ in $\left(c_{0},+\infty\right)$ such that $T_{\tilde{c}}>1$ and

$$
w_{\tilde{c}}^{n+1}(1)=\frac{2}{\tilde{c}(n-1)^{2}} S_{\tilde{c}}^{-1}\left(\sqrt{\frac{2}{\tilde{c}}}\right),
$$

where $S_{c}:[0,1] \rightarrow\left[0, S_{c}(1)\right]$ is defined as

$$
S_{c}\left(1-w_{c}^{n-1}(t)\right):=\int_{0}^{1-w_{c}^{n-1}(t)} \frac{1}{h^{\frac{1}{2}}(1-h)^{\frac{n-3}{2(n-1)}}} \mathrm{d} h,
$$

for $t$ in $\left[0, T_{c}\right]$.

Proof It follows from the proof of Theorem 2.7 that if $c>c_{0}$ then there exists $w_{c}(t)$ classical solution of (6.15) in $\left[0, T_{c}\right]$, with $T_{c}>1$.

Now we define $F:\left(c_{0},+\infty\right) \rightarrow \mathbb{R}$ as

$$
F(c)=w_{c}^{n+1}(1)-\frac{2}{c(n-1)^{2}} S_{c}^{-1}\left(\sqrt{\frac{2}{c}}\right) .
$$

It is obvious that $w_{c}(t)$ is continuous on $\left(c_{0},+\infty\right)$ for every $t$ in $\left[0, T_{c}\right)$, so that $F$ is continuous. Fix $c_{0}<c_{1}<c_{2}$. Recalling that

$$
T_{c}=\sqrt{\frac{\pi c}{2}} \frac{\Gamma\left(\frac{1}{2}+\frac{1}{n-1}\right)}{\Gamma\left(\frac{n}{n-1}\right)},
$$

we deduce $T_{c_{1}}<T_{c_{2}}$. Moreover, we state that $w_{c_{1}}(t)<w_{c_{2}}(t)$ for every $t$ in $\left(0, T_{c_{1}}\right]$. Indeed, since $-w_{c_{1}}^{\prime \prime}(t)>-w_{c_{2}}^{\prime \prime}(t)$ near $t=0$ and using the initial conditions, we obtain that $w_{c_{1}}(t)<w_{c_{2}}(t)$ near $t=0$. If, by contradiction, there exists $s$ in $\left(0, T_{c_{1}}\right)$ such that $w_{c_{1}}(s)=w_{c_{2}}(s)$ we have that $w_{c_{1}}^{\prime}(s) \geq w_{c_{2}}^{\prime}(s)$. We know, by (6.2), that

$w_{c_{1}}^{\prime}(s)=-\sqrt{\frac{2}{(n-1)^{2} c_{1}}}\left(w_{c_{1}}^{1-n}(s)-1\right)^{\frac{1}{2}}<-\sqrt{\frac{2}{(n-1)^{2} c_{2}}}\left(w_{c_{1}}^{1-n}(s)-1\right)^{\frac{1}{2}}=w_{c_{2}}^{\prime}(s)$,

which is a contradiction. Hence, we have that $w_{c}(t)$ is monotone increasing in $c$. This implies that $F$ also is monotone increasing in $c$. By letting $c$ tend to the boundary of $\left(c_{0},+\infty\right)$ and recalling that

$$
\lim _{c \rightarrow c_{0}} w_{c}^{n+1}(1)=0 \text { and } \lim _{c \rightarrow+\infty} w_{c}^{n+1}(1)=1
$$


we deduce

$$
\lim _{c \rightarrow c_{0}} F(c)=-\frac{2}{c_{0}(n-1)^{2}} S_{c_{0}}^{-1}\left(\sqrt{\frac{2}{c_{0}}}\right)<0 \text { and } \lim _{c \rightarrow+\infty} F(c)=1 .
$$

Applying Bolzano's theorem, we obtain that there exists $\tilde{c}$ such that $F(\tilde{c})=0$, that is, (6.16). Since $F$ is monotone increasing, $\tilde{c}$ is unique.

\section{Open problems}

We are now studying the nonexistence of positive solutions of (2.8) in the $N$-dimensional case with $f$ only nonnegative. More precisely, we assume that $f$ is a nonnegative $L^{\infty}(\Omega)$ function and that there exists $\omega \subset \subset \Omega$ such that $f=0$ in $\Omega \backslash \omega$ and such that for every $\omega^{\prime} \subset \subset \omega$ there exists $c_{\omega^{\prime}}>0$ such that $f \geq c_{\omega^{\prime}}$ in $\omega^{\prime}$.

We observe that from Remark 6.1 it follows that $u$, given by Theorem 2.7, is a classical solution of

$$
\left\{\begin{array}{l}
-u^{\prime \prime}=0 \quad \text { in }(-2,-1) \cup(1,2) \\
u( \pm 1)=1 \\
u( \pm 2)=0
\end{array}\right.
$$

Our conjecture is that it is true also for $N>1$. More precisely, we think that the following result holds.

Conjecture 7.1 Let $u$ be the function given by Theorem 2.2, with $M(x) \equiv I$. Then, $u$ is a classical solution of

$$
\left\{\begin{aligned}
-\Delta u=0 & \text { in } \Omega \backslash \bar{\omega} \\
u=1 & \text { on } \partial \omega \\
u=0 & \text { on } \partial \Omega .
\end{aligned}\right.
$$

With a similar idea, we think that Theorem 2.7 holds for $N>1$.

Conjecture 7.2 Let $u_{n}$ be the solution of (2.3) given by Theorem 3.1, with $M(x) \equiv I$. Let $\left\{v_{n}=\frac{u_{n}^{n+1}}{n+1}\right\}$ be the sequence of solutions of (2.6). Then, $\left\{v_{n}\right\}$ is bounded in $W_{0}^{1,2}(\Omega) \cap$ $L^{\infty}(\Omega)$, so that it converges, up to subsequences, to a bounded nonnegative function $v$. Moreover, $v$ is a weak solution of

$$
\left\{\begin{aligned}
-\Delta v+\frac{|\nabla v|^{2}}{v} & =f \quad \text { in } \omega \\
v & =0 \text { on } \partial \omega
\end{aligned}\right.
$$

and $v \equiv 0$ in $\Omega \backslash \omega$.

\section{References}

1. Abramowitz, M., Stegun, I.A.: Handbook of Mathematical Functions with Formulas, Graphs, and Mathematical Tables. National Bureau of Standards Applied Mathematics Series 55. Dover, New York (1965) 
2. Arcoya, D., Barile, S., Martínez-Aparicio, P.J.: Singular quasilinear equations with quadratic growth in the gradient without sign condition. J. Math. Anal. Appl. 350, 401-408 (2009)

3. Arcoya, D., Carmona, J., Leonori, T., Martínez-Aparicio, P.J., Orsina, L., Petitta, F.: Existence and nonexistence of solutions for singular quadratic quasilinear equations. J. Differ. Equ. 246, 4006-4042 (2009)

4. Arcoya, D., Martínez-Aparicio, P.J.: Quasilinear equations with natural growth. Rev. Mat. Iberoam. 24, 597-616 (2008)

5. Arcoya, D., Segura de León, S.: Uniqueness of solutions for some elliptic equations with a quadratic gradient term. ESAIM Control Optim. Calc. Var. 16, 327-336 (2010)

6. Boccardo, L.: Dirichlet problems with singular and gradient quadratic lower order terms. ESAIM Control Optim. Calc. Var. 14, 411-426 (2008)

7. Boccardo, L., Casado-Díaz, J.: Some properties of solutions of some semilinear elliptic singular problems and applications to the G-convergence. Asymptot. Anal. 86, 1-15 (2014)

8. Boccardo, L., Orsina, L.: Semilinear elliptic equations with singular nonlinearities. Calc. Var. PDEs 37, 363-380 (2010)

9. Boccardo, L., Orsina, L., Porzio, M.M.: Existence results for quasilinear elliptic and parabolic problems with quadratic gradient terms and sources. Adv. Calc. Var. 4, 397-419 (2011)

10. Bougherara, B., Giacomoni, J.: Some regularity results for a singular elliptic problem. Dyn. Syst. Differ. Equ. Appl. Proc. AIMS 2015, 142-150 (2015)

11. Bougherara, B., Giacomoni, J., Hernández, J.: Existence and regularity of weak solutions for singular elliptic problems. In: Proceedings of the 2014 Madrid Conference on Applied Mathematics in Honor of Alfonso Casal, Electronic Journal of Differential Equations, Conference, vol. 22, pp. 19-30 (2015)

12. Brandolini, B., Chiacchio, F., Trombetti, C.: Symmetrization for singular semilinear elliptic equations. Ann. Mat. Pura Appl. 4, 389-404 (2014)

13. Canino, A., Montoro, L., Sciunzi, B.: The moving plane method for singular semilinear elliptic problems. Nonlinear Anal. 156, 61-69 (2017)

14. Canino, A., Sciunzi, B.: A uniqueness result for some singular semilinear elliptic equations. Commun. Contemp. Math. 18, 1550084 (2016)

15. Carmona, J., Leonori, T.: A uniqueness result for a singular elliptic equation with gradient term. Proc. R. Soc. Edinb. Sect. A 148, 983-994 (2018)

16. Carmona, J., Martínez-Aparicio, P.J., Rossi, J.D.: A singular elliptic equation with natural growth in the gradient and a variable exponent. NoDEA Nonlinear Differ. Equ. Appl. 22, 1935-1948 (2015)

17. Chipot, M., De Cave, L.M.: New techniques for solving some class of singular elliptic equations. Rend. Lincei Mat. Appl. 29, 487-510 (2018)

18. Coclite, G.M., Coclite, M.M.: On a Dirichlet problem in bounded domains with singular nonlinearity. Discrete Contin. Dyn. Syst. 33, 4923-4944 (2013)

19. Crandall, M.G., Rabinowitz, P.H., Tartar, L.: On a Dirichlet problem with a singular nonlinearity. Commun. Partial Differ. Equ. 2, 193-222 (1977)

20. Croce, G.: An elliptic problem with degenerate coercivity and a singular quadratic gradient lower order term. Discrete Contin. Dyn. Syst. Ser. S 5, 507-530 (2012)

21. Dall'Aglio, A., Orsina, L., Petitta, F.: Existence of solutions for degenerate parabolic equations with singular terms. Nonlinear Anal. 131, 273-288 (2016)

22. De Cave, L.M., Durastanti, R., Oliva, F.: Existence and uniqueness results for possibly singular nonlinear elliptic equations with measure data. NoDEA Nonlinear Differ. Equ. Appl. 25, 18 (2018)

23. del Pino, M.: A global estimate for the gradient in a singular elliptic boundary value problem. Proc. R. Soc. Edinb. Sect. A 122, 341-352 (1992)

24. Esposito, F., Farina, A., Sciunzi, B.: Qualitative properties of singular solutions to semilinear elliptic problems. J. Differ. Equ. 265, 1962-1983 (2018)

25. Giachetti, D., Martínez-Aparicio, P.J., Murat, F.: Definition, existence, stability and uniqueness of the solution to a semilinear elliptic problem with a strong singularity at $u=0$. Ann. Sc. Norm. Super. Pisa Cl. Sci. 18, 1395-1442 (2018)

26. Giachetti, D., Martínez-Aparicio, P.J., Murat, F.: On the definition of the solution to a semilinear elliptic problem with a strong singularity at $u=0$. Nonlinear Anal. 177, 491-523 (2018)

27. Giachetti, D., Petitta, F., Segura de León, S.: A priori estimates for elliptic problems with a strongly singular gradient term and a general datum. Differ. Integr. Equ. 26, 913-948 (2013)

28. Lair, A.V., Shaker, A.W.: Classical and weak solutions of a singular semilinear elliptic problem. J. Math. Anal. Appl. 211, 371-385 (1997)

29. Lazer, A.C., McKenna, P.J.: On a singular nonlinear elliptic boundary-value problem. Proc. Am. Math. Soc. 111, 721-730 (1991)

30. Leoni, F.: Nonlinear elliptic equations in $\mathbb{R}^{N}$ with "absorbing" zero order terms. Adv. Differ. Equ. 5, 681-722 (2000) 
31. Littman, W., Stampacchia, G., Weinberger, H.F.: Regular points for elliptic equations with discontinuous coefficients. Ann. Sc. Norm. Sup. Pisa 17, 43-77 (1963)

32. Martínez-Aparicio, P.J.: Singular Dirichlet problems with quadratic gradient. Boll. Unione Mat. Ital. 2, 559-574 (2009)

33. Oliva, F.: Regularizing effect of absorption terms in singular problems. J. Math. Anal. Appl. 472, 11361166 (2019)

34. Oliva, F., Petitta, F.: Finite and infinite energy solutions of singular elliptic problems: existence and uniqueness. J. Differ. Equ. 264, 311-340 (2018)

35. Orsina, L., Petitta, F.: A Lazer-McKenna type problem with measures. Differ. Integr. Equ. 29, 19-36 (2016)

36. Porru, G., Vitolo, A.: Problems for elliptic singular equations with a quadratic gradient term. J. Math. Anal. Appl. 334, 467-486 (2007)

37. Stampacchia, G.: Le problème de Dirichlet pour les équations elliptiques du seconde ordre à coefficients discontinus. Ann. Inst. Fourier (Grenoble) 15, 189-258 (1965)

38. Stampacchia, G.: Équations elliptiques du second ordre à coefficients discontinus, Séminarie de Mathématiques Supérieures 16, Montréal (1966)

39. Stuart, C.A.: Existence and approximation of solutions of nonlinear elliptic equations. Math. Z. 147, 53-63 (1976)

40. Sun, Y., Zhang, D.: The role of the power 3 for elliptic equations with negative exponents. Calc. Var. PDEs 49, 909-922 (2014)

41. Xia, L., Yao, Z.: Existence, uniqueness and asymptotic behavior of solutions for a singular parabolic equation. J. Math. Anal. Appl. 358, 182-188 (2009)

42. Zhou, W.: Existence and multiplicity of weak solutions for a singular semilinear elliptic equation. J. Math. Anal. Appl. 346, 107-119 (2008)

43. Zhou, W., Wei, X., Qin, X.: Nonexistence of solutions for singular elliptic equations with a quadratic gradient term. Nonlinear Anal. 75, 5845-5850 (2012)

Publisher's Note Springer Nature remains neutral with regard to jurisdictional claims in published maps and institutional affiliations. 